
QL
524.2
P7
ytom.
$(595.7$
ENT。
DIE
$p q 22$

\title{
AMITOTISCHE KERNTHEILUNG
}

\section{IN DEN \\ OVARIEN DER HENIITEREN.}

\section{INAUGURAL-DISSERTATION}

VORGELEGT DER

(HOHEN PHILOSOPHISCHEN FAKULTÄT

DER

UNIVERSITÄT MARBURG)

BEHUFS

ERLANGUNG DER PHILOSOPHISCHEN DOKTORWÜRDE

VON

\section{FRANZ PREUSSE}

ÀUS BRAUNSCHWEIG.

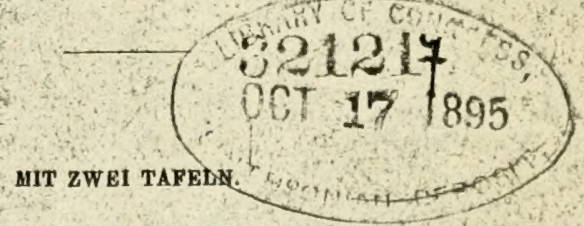

M A R B U R 1895. 


Ent.

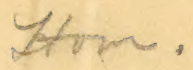

\title{
57.4
}

DIE

\section{AMITOTISCHE KERNTHEILUNG}

\author{
IN DEN

\section{OVARIEN DER HEIIIPTEREN.}

\section{INAUGURAL-DISSERTATION}

VORGELEGT DER

HOHEN PHILOSOPHISCHEN FAKULT ÄT

DER

UNIVERSITÄT MARBURG

BEHUFS

ERLANGUNG DER PHILOSOPHISCHEN DOKTORWÜRDE

VON

\section{FRANZ PREUSSE}

AUS BRAUNSCHWEIG.

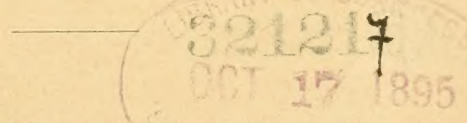

MIT ZWEI TAFELN.

M A R B U R G 1895. 
Von der Fakultät als Dissertation angenommen am 24. Januar 1895. Fakn

(Separat-Abdruck aus: Zeitschrift für wissensch. Zoologie. Bd. LIX. 2. Heft.) 


\section{DEN MANEN}

\section{S E I N E S V A T E R S}

IN DANKBARKEIT GEWIDMET

VOM

VERFASSER. 
Die Frage nach dem Vorkommen und der Bedeutung der amitotischen Kerntheilung im Thierreich ist in den letzten Jahren Gegenstand zahlreicher Untersuchungen gewesen. Ausführliche Berichte über dieselben sind von berufenster Seite, nämlich von Fumming in den Ergebnissen morphologischer Forschung (1893 u. 1894) gegeben worden, so dass ich bezüglich der Litteratur dieses Gegenstandes auf jene eingehende Darstellung verweisen kann. Aus Flemming's Zusammenfassung, in welcher auch über Wesen und Bedeutung der amitotischen Kerntheilung gehandelt wird, geht hervor, dass trotz der umfangreichen Litteratur, die bereits über die Amitose vorhanden ist, die Anschauungen über ihre Bedeutung durchaus noch nicht genügend geklärt sind. Während einige Forscher geneigt sind, der Amitose eine mehr oder minder große Bedeutung zuzuschreiben, halten andere sie für weniger wichtig, ja sprechen sie wohl gar für einen »degenerativen Vorgang“ an. Vor Allem ist das Verhältnis der amitotischen zur mitotischen Kerntheilung noch völlig in Dunkel gehüllt. Bei dieser Lage der Sache erscheint es daher von Wichtigkeit, diejenigen Fälle genau zu untersuchen, in denen die Amitose mit Sicherheit zu beobachten ist und ein gewöhnliches Vorkommen darstellt, wobei den Fällen besondere Aufmerksamkeit zu schenken ist, in welchen die Amitose in Verbindung mit einer reichen Vermehrung der Zellen auftritt. Dies ist besonders wichtig in Hinblick auf die in neuerer Zeit zu größerer Geltung gelangte Auffassung, nach welcher die Zellen, welche sich 
auf amitotischem Wege theilen, allmählichem Untergang geweiht sind. Bei Ziegler tritt diese Auffassung der amitotischen Theilung am prägnantesten hervor: "Amitose tritt hauptsächlich in Zellen auf, die in Folge besonderer Specialisirung einer intensiven Assimilation, Sekretion oder Exkretion vorstehen, ferner in alternden, abgenutzten Geweben und folglich auch da, wo Zellen nur eine vorübergehende Bedeutung haben. Den Mitosen gegenüber haben die Amitosen einen degenerativen Charakter. "

$\mathrm{Zu}$ denjenigen Objekten, bei welchen die amitotische Kerntheilung sehr häufig ist und allem Anschein nach eine besondere Rolle spielt, gehören nach den Angaben von Konschelt die Ovarien der Insekten und speciell der Hemipteren. Im Ovarialepithel und in der Endkammer fand er zahlreiche Zellen, welche zwei Kerne enthalten und welche bei der großen Verbreitung, die sie im Ovarium besitzen, kaum anders, als in der amitotischen Theilung begriffen aufgefasst werden konnten, zumal auch Zellen mit einem einzigen, aber eingeschnürten Kern vielfach vorhanden waren. Derartige Zellen mit einem runden oder anscheinend in Theilung begriffenen Kern, sowie besonders auch mit zwei Kernen kommen in den Ovarien der Hemipteren mit großer Regelmäßigkeit und außerordentlich häufig vor. Es schien daher lohnend, diesen, von Korschelt in seinen Arbeiten über die Insektenovarien nur kurz berührten Verhältnissen weiter nachzugehen. Desshalb folgte ich sehr gern der mir von Herrn Professor Korschelt gegebenen Anregung, diese Vorgänge zum Gegenstand einer eingehenden Untersuchung zu machen.

Es sei mir an dieser Stelle gestattet, meinem hochverehrten Lehrer für das fortdauernde rege Interesse, welches er jederzeit für meine Arbeiten hegte und für die stets gütige, liebenswürdige Unterweisung meinen verbindlichsten Dank auszusprechen.

\section{Material und Mothode.}

Die Untersuchungen erstreckten sich auf mehrere Vertreter aus dem Stamme der Insekten. Am eingehendsten wurden die Hemipteren, die nach der obigen Angabe besonders geeignet sind, und speciell Nepa cinerea studirt.

Die Herausnahme der Eierstöcke erfolgte beim lebenden oder durch Chloroform betäubten Thier möglichst rasch mit oder ohne Anwendung physiologischer Kochsalzlösung.

Als Fixirungsmittel kamen zur Anwendung koncentrirte, kalte Sublimatlösung (5-10 Minuten), sowie Chromosmiumessigsäure nach 
Flemming und nach Carnor, endlich Pikrinschwefelsäure nach KlenexBERG (8 Stunden).

Zur Darstellung der Centrosome wurde noch Platinchlorid mit Nachbehandlung von Holzessig angewendet.

Die Färbung der Eiröhren geschah mit Boraxkarmin und Hämatoxylin in toto und auf Schnitten. Wiihrend die jüngeren Stadien der Eibildung bei Anwendung der Schnittmethode ohne Weiteres brauchbare Bilder lieferten, wurde versucht, den mittleren und älteren mehr gewölbten Eiern dadurch eine für das Schneiden geeignetere Form zu geben, dass sie zwischen Objektträger und beschwertem Deckglas einem leichten Druck ausgesetzt wurden. Tangentiale Schnitte konnten hierdurch in etwas größerer Ausdehnung gewonnen werden. Es versteht sich von selbst, dass bei dieser Behandlung die nöthige Vorsicht angewandt werden musste.

Recht gute Resultate lieferten die Versuche, das Ovarialepithel mechanisch vom Dotter abzulösen, welches Verfahren sich vom elften Eifach an ausführen ließ. Aber auch hier boten sich Hindernisse mancherlei Art dar, bestehend in der trotz langer Einwirkung von absolutem Alkohol noch immer morschen Konsistenz der Follikel, der naturgemäßen Wölbung, der Verklebung des Epithels mit dem Chorion und besonders in den Anhängen der Dotterpartikelchen, von denen die Eihülle nur sehr schwer durch Nadel und Pinsel befreit werden konnte, ohne zu zerreißen. Iläufig blieben dabei nur winzige Fetzen übrig. War bei den Eiern das Chorion bereits gebildet, so musste dasselbe vom Epithel abgelöst werden, weil sonst das Präiparat zu wenig durchsichtig wurde. Leichter ließ sich das Epithel in den Stadien präpariren, in denen die Ausbildung des Chorions noch nicht erfolgt war, jedoch wirkte nun die geringe Größe der Eier störend. Immerhin erwies sich dieses Verfahren trotz der genannten Ilindernisse als recht brauchbar.

Die Fürbung der so erhaltenen Struckchen des Ovarialepithels geschah erstens durch Boraxkarmin; bei den mittels Pikrinschwefelsäure fixirten Präparaten jedoch durch Pikrokarmin; drittens kam bei Chromosmiumessigsäure-Vorbehandlung zumeist Ilämatoxylin zur Anwendung. Schließlich wurde auch bei letzterer Konservirung die Färbung durch Gentianaviolett versucht.

Die besten Resultate lieferten die Chromosmiumessigsäure-Präparate mit nachfolgender Hämatoxylinanwendung. 


\section{Untersuchungen an Nepa cinerea.}

\section{Vorbemerkungen.}

Die Untersuchung hatte sich auf verschiedene Theile der Ovarien zu erstrecken, wesshalh ich eine kurze Schilderung des Baues derselhen vorausschicken muss.

Jedes der beiden Ovarien von Nepa cinerea besteht aus einem Komplex von fünf Eiröhren, die nach hinten konvergiren. Jede Eirohre setzt sich an dem vorderen Ende in den Endfaden, der sie an das Ruckengefaß befestigt, fort, nach dem anderen verlängert sie sich zum Ëröhrenstiel. Dieser mundet in den Eierkelch, welcher in den gemeinsamen Eileiter übergeht. Beide Eileiter treten dann zum unpaaren Stamm desselben zusammen.

Die :ufere Bekleidung des Endfadens und jeder Eiröhre bildet die Peritonealhülle. Die die Eiröhren umspinnenden Tracheen stellen Verbindungen derselben unter einander und mit benachbarten Theilen her.

Jede Eiröhre bestelıt aus dem Endfaden, der Endkammer und der eigentlichen Eiröhre, d. h. dem umfangreichen Abschnitt, welcher die in der Ausbildung begriffenen Eier enthält.

Die Endkammer, welche von Will und Konscuect eingehend beschriehen wurde, ist ein keulenförmiges Gebilde, welches sich nach vorn in den kndfaden fortsetzt und nach hinten zu in die eigentliche Eiröhre äbergeht. Erfüllt ist die Endkammer zum größten Theil ron einer großen Menge von umfangreichen Zellen. Diese sind zumeist an der Spitze der Endkammer kleiner und nehmen nach hinten an Umfang zu. Es sind dies die Zellen, welche Wrut als Ooblasten bezeichnet, während Konschelt sie als Nährzellen, Wielowiejshi als Dotterbildungszellen anspricht. Die letzteren Auffassungen dürften wegen der von Korscurt eingehend geschilderten Auflösung der genannten Zellen und des Ilereintretens der Verbindungsstränge in die Mitte der Lndkammer jedenfalls die richtigen sein.

Der von mir im Einklange mit versehiedenen Autoren für das Keimlager gehaltene Theil der Eiröhre bildet den untersten Mbschnitt der Endkammer. Nach oben zu stöt er an den Nährzellenkomplex, auf der anderen Seite begrenzen ihn die jüngsten Ëficher. Von diesen reiht sich nun Eifach an Eifach, gradatim an Größe zunchmend. Zuniichst sitzen die Eificher noch mit breiter Basis einander auf, bald jedoch zeigen sich Ëinsehnürungen, die je weiter nach unten gelegen, um so stirker werden. In den letzten drei his vier Eifichern verschmitlert sich dic verbindende P'artie zu einem Strange. Neben dem Auftreten 
dieser Einschnürungen geht nun auch zugleich die Aushildung des ron Konscmelt beschriebenen Aufsatzes einher, welcher die Abscheidung der eigenthümlichen, das Li von Nepa auszeichnenden "Strahlen " besorgt. Die Bildung des Aufsatzes, welche ich wegen der hier besonders reichen Zellenvermehrung genauer verfolgte, verläuft so, wie dies von Konscielt beschrieben wurde. Der Aufsatz wird vom achten bis neunten Eifach an deutlich. Ich bezeichne die erste Eianlage, welche die ganze Breite der Eiröhre einnimmt, mit Nr. I und zähle daun weiter nach rückwärts. An das letzte und größte Ei reiht sich ebenfalls mit der genannten Verbindung oder aber auch direkt der vom ausgestoßenen Ei verlassene Follikel, der zugleich den Anfangstheil des Eiröhrenstiels vorstellt. Der letztere ist ein langes, schlauchförmiges Gebilde mit vielen Krausen, Faltungen und $\Lambda$ usbuchtungen, in dessen unteren Theil die chitinigen Strahlen des ausgestoßenen Eies hineinreichen können. Dieses bildet den Beschluss der Eierfolge, und an dasselbe reiht sich der Leitungsweg, so wie schon oben erwähnt wurde.

\section{Die Kerntheilungen im Ovarialepithel.}

A. Die alteren Eifächer.

Zunächst hehandle ich das Epithel der etwas ailteren Lier, bei denen es sich auf die Eingangs erwaihnte Weise durch Abpinseln darstellen lieb.

Bei oberflichlicher Betrachtung erscheint es aus platten, polygonalen Zellen zusammengesetzt, deren Grenzen je nach der Vorbehandlung mehr oder weniger deutlich sind. Jede dieser Zellen enthilt einen oder zwei Kerne, die ihrerseits mit einem sehr deutlichen Nucleolus versehen sind, oder auch zwei Nucleolen besitzen (Taf. XIX).

Die nähere Untersuchung stellt nun Folgendes fest:

Die Zellgrenzen erscheinen als gerade Linien oder sie sind leicht gekrümmt. Zuweilen stoßen sie unter scharfem Winkel zusammen, so dass ziemlich regelmißBige Fünf- oder Sechsecke entstehen. Nicht selten jedoch ist eine oder die andere Ecke abgerundet.

Beim geraden Verlauf der Grenzen treten die Intercellularriume zuriack; jedoch erseheinen solche Zwischenraiume in Folge des Aneinandertretens der gekrummten Grenzlinien benachbarter Zellen. Diese Zwischenraume sind oft recht umfangreich und müssen wohl zum größten Theil als Kunstprodukte aufgefasst werden.

Die Zellen beherbergen, wie schon kurz erwihnt, einen oder zwei Kerne, die den größeren Theil der Zelle erfüllen. In Folge dessen bleibt das Protoplasma auf eine im Ganzen ziemlich schmale Randzone beschränkt. Das Protoplasma erschien unter Anwendung der Vergrößerungen, bei denen die Zeichnungen angefertigt wurden, ziemlich 
homogen. Unter Zuhilfenahme von stärkeren Systemen war jedoch eine undeutliche Körnelung zu erkennen, und je nach der Menge dieser Körnchen zeigte es sich bald mehr hyalin, bald dunkler. Bei den mit Chromosmiumessigsiiure fixirten Objekten nahm es die Farbe wenig an. Das umgekehrte Verhalten hatte bei der Fixirung mit Sublimat statt. In diesem Falle firbte sich das Protoplasma stärker, als die Kerne, wie auch aus den Zeichnungen Fig. 10 und 12 zu crkennen ist, wïhrend das erstgenannte Verhalten sich zum Beispiel aus den Fig. 3-8 ergiebt.

Die nun folgende Schilderung wird sich zunächst auf die Kerne selbst erstrecken. In einem gesonderten, daran schließenden $\mathrm{Ab}$ schnitte werde ich die Verhältnisse der Kernkörper behandeln.

Falls die Kerne in der Einzahl vorhanden sind, zeigen sie im Allgemeinen eine rundliche oder ovale Gestalt (Fig. $2 c, 7 b$ ). Zuweilen besitzen sie Ausbuchtungen, die mehr oder weniger stark hervorragen und sich in kleine spitze Fortsätze verlängern können. Im Ganzen passen sich die Kerne der Form der zugehörigen Zellen ziemlich gut an.

Die Größe der Kerne variirt, sowie die der Zellen, innerhalb weiter Grenzen. Jedoch ist oft eine gewisse Gesetzmäßigkeit in den Größenverhältnissen zu erkennen. So finden sich Kerne, die an Volumen der Summe zweier Kerne einer benachbarten Zelle ungefahr gleich kommen Fig. 15a). Mit gleicher Häufigkeit kommen Paare neben einander liegender Zellen mit je nur einem Kern vor (Fig. 2 c $c$ und (l), die zusammengenommen einer in der Nähe befindlichen Zelle mit zwei solchen Kernen entsprechen, wie die Figuren ohne Weiteres erkennen lassen. Jeder dieser beiden Kerne stimmt dann seinerseits nahezu an Größe mit einem der beiden Einzelkerne überein. Diese Unterschiede in der Größe sind jedenfalls der Ausdruck davon, dass die kleineren Kerne durch Theilung eines größeren Kernes entstanden. Die erstgenannten, großen Kerne (z. B. Fig. $3(a$ und $15 \%$ ) stehen wahrseheinlich vor der Theilung, wie die späteren Ausführungen zeigen werden. Es wird sich ergeben, dass zumal auch die Gestaltsverhälnisse der Kernkörper für diese Auffassung sprechen.

Mil einer je nach den Altersstadien, auf welchen sich die Eificher befinden, wechselnden IIaufigkeit sind die Kerne in den Zellen in doppelter Anzahl vorhanden. Die Größe der in je einer Zelle enthaltenen beiden Kerne ist ebenfalls mannigfachen Schwankungen unterworfen. Immer aber erfüllen auch sie den größeren Theil der Zelle. Innerhalb einer und derselben Zelle jedoch kommen die beiden Kierne einander an Volumen ungefahr gleich. Die verschiedenen Abbildungen der Tafel XIX erläutern diese Verhältnisse. 
Wenngleich nun auch häufig cine Konformität in den Grölsenverbältnissen der Zellen mit zwei Kernen eines Bezirks besteht, derart, dass ihr Volumen ungefahr gleich ist, sind andererseits auch solche Stellen nicht selten, an denen ihr Umfang erheblich differirt.

Eine Zelle mit zwei Kernen kann einer eben solchen direkt benachbart sein, die erstere um das Doppelte und Mehrfache ibbertrillt (Fig. 6 (l). Dieses Verhalten setze ich cinerseits auf Rechnung der Theilung, andererseits schreibe ich es mit WiLL Wachsthumsvorgingen zu.

Was nun die Größenverhältnisse der Kerne in Rücksicht auf die Altersstadien der Eifächer anbetrifft, so gilt, dass je älter der Follikel ist, er auch um so größere Kerne aufweist.

Die gegen die Zellgrenzen gerichteten Ränder der Kerne sind im Allgemeinen mehr oder minder stark nach außen gekrümmt. Die gegen einander gekehrten Begrenzungen sind im Epithel der zuerst mittels Abpinselns behandelten Eifficher, 11-13, ziemlich gerade Linien, wenngleich auch schon hier Abweichungen im Sinne des sogleich zu schildernden Verhaltens der anderen Eifächer vorkommen. In den ätteren Eifächern, 14 und folgende, erscheinen die gegen einander gekelsten Grenzen der beiden Kerne mehr oder weniger stark eingebuchtel. Mittels bogenförmiger Krtämmung geht die innere Begrenzung der Kerne in die außere über, doch kann sich der Kern an dieser Stelle auch ziemlich scharf zuspitzen.

Durch das geschilderte Verhalten kommen mannigfache Formen von Kernen zu Stande. Vielfach ist die parallel verlaufende Achse beider Kerne die längere; erscheint dann gleichzeitig das eine oder auch das andere Ende der Kerne spitz ausgezogen, so zeigen sich dieselben halbmondförmig gestaltet (Fig. $4 e, 10$ l). Bei stïrkerer Krïmmung des konvexen Randes wird dieser schließlich halbkreisförmig.

Durch stïkere oder schwächere Verjüngung eines oder beider Enden, durch den versehiedenen Grad der Kírúmmung des inneren und äußeren Saumes kommen die mannigfachsten Formen der Kerne zu Stande, worauf ich nicht besonder's eingehe, sondern auf die verschicdenen beigegebenen Figuren verweise.

Zwischen beiden Kernen bleibt je nach dem näheren oder weiteren Abstand derselben und je nach dem Verlauf der inneren Rimcler ein verschieden großer, resp. versehieden gestalteter Zwischenraum, der gewöhnlich dunkler, als die kerne selhst erscheint (Fig. it und 8). An den Enden der Kerne geht er allmihlich in das Protoplasma der Zelle uher. In dieser Stelle setzt sich übrigens zuweilen das Plasma des Zwischenraums ziemlich scharf von dem ubrigen Zellplasma ab. Mög- 
licherweise hangt dies mit der vorhergegangenen Theilung der kerne zusammen. Ich werde weiter unten noch darauf zuridckzukommen haben.

Was die Gestalt des Zwischenraumes anbetrifft, so zeigt er in manchen Faillen in seiner ganzen Erstreckung die nämliche Breite Fir. 5 ('). In der Mehrzahl der Faille jedoch ist er entsprechend den cinander zugewandten, konkaven Kontouren der Kerne in der Hitte verbreitert (Fig. $11 e, 14 f)$.

Eingestreut zwischen die Zellen mit Kernen in Ein- und Zweizahl finden sich andere, welche ganz hesondere und für die Auffassung der Zellen mit zwei Kernen wichtige Verhälnisse zeigen. Manche Kerne weisen nämlich eine theilweise oder ganz über sie hin verlaufende Linie auf, welche oftmals eben nur angedeutet und schwer erkennbar ist. In den Figuren 1 und $8 c, 11 f$ und $14 e$ ist dies Verhalten wiedergegeben. Ich kann dasselbe nicht anders aufassen, als dass es sich hier um die amitotische Theilung der betreffenden Kerne handelt. Der Kern wird durch eine Furche in zwei Irailften getheilt. Die beiden Theilprodukte sieht man schon mehr oder weniger deutlich alggegrenzt. Im weiteren Verlaufe vertieft sich die Rinne mehr und mehr Fig. 14 ct), so dass man von oben hetrachtet zwei neben einander liegende Theilstiucke sieht, welche unten auf einer noch in voller liontinuitit befindlichen gemeinsamen Basis aufsitzen. Der Umfang der letzteren nimmt mit dem Fortschreiten des Theilungsprocesses allmaihlich ab und schließlich resultiren zwei Hälften des Kernes.

Lin anderer mit großer Häuligheit vorkommender Modus der Theilung ist der, bei welchem dieselbe von einer Seite her einsetzt. Auch alle Stadien dieses Processes sind ohne große Schwierigheit aufufinden. ligy. \& /l zeigt die erste P'hase desselben, indem der Rand des liernes an einer Stelle eine seichte Einkerbung erlitten hat. Fig. $6 b, 13 c^{\prime}, 1 c^{\prime}$ und / reprösentiren die folgenden Stadien. Die einschneidende Furche hat sich zu einem Spalte ausgebildet, der versehieden weit in das Innere der hermmasse vorgedrungen ist. Oft ist er am Rande schmiller als im Inneren des liernes. Wie der oben beschriebene Raum zwischen den heiden kiernen hebt er sich ebenfalls durch seine dunkle Firbung von dem ibrrigen Protoplasma ab. Der Effekt einer derartigen Theilung ist nun auf diesem Punkte der, dass eine Figur entsteht, welche einem Ilufeisen :ihnelt. Der "Bogen " desselben wird durch die zusammenhängende, noch ungetheilte Partie des Kernes dargestellt, während die "Sichenkel" den bereits getheilten Kernabschnitten gleichkommen. Das Endergebnis der Theilung ist auch hier der Zerfall de's Kernes in zwei Theilstücke. 
Die beiden geschilderten Theilungsvorginge können sich kombiniren (Fig. $3 c, 14 \mathrm{~g}$ ). Dann erfolgt die Durchschnürung gleichzeitig ron oben und ron der Seite her. Hierbei künnen beide Processe ziemlich gleichzeitig verlaufen oder der eine kann dem andereu etwas vorausgehen.

Seltener zeigen die Kerne ein Verhalten, bei dem die Einschnurungen an zwei gegenüberliegenden Punkten gleichzeitig einsetzten. Dieses beobachtet man besonders bei langgestreckten Kernen (Fig. 12 a, 14h). Hieraus resultirt dann eine sogenannte Biskuit- oder falls dieser Process in höherem Maßje fortgeschritten ist, eine Ilantelform. Allein auch hier kanu der Theilungsprocess gleichzeitig in der früher geschilderten Weise von oben her einsetzen. Es erührigt noch, einen letzten Modus der Theilungen zu schildern.

Die Theilung kann nämlich auch derart vor sich gehen, dass zulerst inmitten des Kernes eine dunkle Partie sichtbar wird. An dieser Stelle war der Kern zunächst dünner geworden, und schließlich batte sich hier eine Öffnung gebildet (Fig. $16 a$ ). Dieses Loch im Kern kann sich nach zwei entgegengesetzten Seiten ausbreiten (Fig. 16\%). Hierdurch bekommt es die Gestalt eines Spaltes (Fig. 16b, 15c). Weitere Folgestadien und zugleich das Ende des Processes zeigen Fig. 11 c, 11/, in denen die korrespondirenden Enden der beiden demnächstigen Paarlinge sich nur noch berühren.

Die Regel, nach welcher immer ein oder zwei Kerne, sowie Theilungsstadien von Kernen in einer Zelle vorhanden sind, erleidet auf Grund einiger, wenn auch verhältnismäßig seltener, Befunde gewisse Modifikationen. In manchen Fällen findet sich nämlich außer dem einen Kern noch ein zweiter, schon wieder in Theilung begriffener; in anderen Fällen sind zwei bereits vollständig getheilte Kerne und ein in Theilung befindlicher vorhanden. Endlich trifft man gelegentlich zwei Paare zusammengehöriger Kerne. Erstgenanntes Verhalten illustrirt die Zelle $b$ in Fig. 7. Dieselbe ist langgestreckt und zeigt ungefahr in der Mitte eine Einschnưrung, ein Anzeichen der bald vollendeten Theilung. Die eine Partie beherbergt einen großen, ovalen Kern, während die andere von einem zweiten erfültt ist, der nach dem oben an erster Stelle geschilderten Typus die amitotische Theilung zu vollziehen im Begriffe steht. Die nächste Phase dieses Processes zeigt Zelle $f$ in Fig. 15. Die Gestalt derselben entspricht del' eben abgehandelten Zelle; nur ist die angebahnte Theilung der Zelle noch nicht ganz so weit vorgeschritten, wie in jenem Falle. Wiederum zeigt sich ein Kern, diesmal mittels Auftreten eines Spaltes, in der direkten Theilung begriffen. Der andere Abschnitt der Zelle dagegen 
weist hereits das Resultat der Theilung, die zwei durch einen dunklen Spalt von einander getrennten Kerne auf.

Wurde in einem anderen ihnlichen Falle sich die Theilung des ersteren Kernes früher vollziehen, als diejenige der Zelle, so müsste eine Zelle resultiren, die also zwei Paare zusammengehöriger Kerne enthielte. Diese zuletzt mitgetheilten Befunde sprechen für eine rasche Weitertheilung der aus einem Mutterkern entstandenen Tochterkerne.

Bezüglich der zuletzt besprochenen Vorgïnge könnte sich die Vermuthung aufdrängen, dass die Abgrenzung zwischen den beiden Abtheilungen der in die Länge gezogenen Zelle (Fig. 7 b, 15/) nur ühersehen worden sei, doch habe ich solche Fiille wiederholt mit Sicherheit beobachtet, und die Zusammengehürigkeit der beiden Abtheilungen der Zelle tritt im Präparat weit deutlicher hervor, als ich dies durch die Zeichnung wiederzugeben vermochte.

Nach Abhandlung der für die Theilung der Kerne maßgebenden Verhïlnisse habe ich jetzt zunächst noch Einiges über den allgemeinen Charakter der Kerne nachzutragen. Bei allen drei beschriebenen Arten, den in Ruhe befindlichen, den in Theilung begriffenen und den bereits getheilten Kernen sind die Kontouren deutlich und meist glatt. In Ausnahmefillen jedoch sind die in Bildung befindlichen, einander zugekehrten Ränder von Kernen, die sich nach dem oben an zweiter Stelle angeführten Modus theilen, mit zahlreichen kleinen Zacken versehen (Fig. $17 \mathrm{e}$ ). Hierdurch wird man an die Angaben und Bilder einiger Autoren erinnert, welche eine derartige Erscheinung als ein Bestreben nach einer Oberflächenvergrößerung ansehen.

Was den feineren Bau der Kerne anbetrifft, so weisen dieselben ein dichtes Netzwerk von Fäden auf. Die letzteren bewirken, dass die Kerne bei nicht schr starker Vergröß Berung granulirt erscheinen.

Noch eine Eigenthümlichkeit von seltenem Vorkommen müchte ich an dieser Stelle erwähnen. Es färben sich nämlich zuweilen bei Chromosmiumessigsiure-Konservirung die Kerne eines Territoriums ungleichmäßig, so dass sich hierdureh Paarlinge und Gruppen von Kernen schon von vorn herein als zusammengehörig zu erkennen geben. Sodann muss ich auf eine andere Erscheinung noch aufmerksam machen, nämlich auf die Änderung des quantitativen Verhältnisses zwischen einund zweikernigen Zellen. Wie wir gesehen haben, halten sie sich auf denjenigen Stadien, die uns die ersten Oberflichenbilder liefern, an Menge ungefahr das Gleichgewicht. Bald jedoch überwiegen die zweikernigen Zellen immer mehr und mehr. In den letzten Eifächern haben sie die Oberhand, wenngleich auch hier bald mehr vereinzelt, 
bald häufiger einkernige Zellen und amitotische Theilungsfiguren noch vorkommen.

Bei den hier angestellten Beobachtungen liegt es nahe, nach der Betheiligung der Centrosome bei den geschilderten Theilungsvorgängen zu fragen. Leider sind jedoch meine diesbezliglichen Untersuchungen negativ ausgefallen. Trotz der Anwendung der für die Hervorhebung der Centrosome vorgeschriebenen Konservirungsmethoden und der dafur empfohlenen Farbstoffe konnte ich ungeachtet meines eifrigen Suchens niemals mit völliger Sicherheit das Vorhandensein von Centrosomen und Attraktionssphären feststellen. Zwar findet sich bäufig im Protoplasma, zuweilen auch deutlich im Kern ein auffallendes, dunkles Korn von einem hellen Hof umsäumt. Dennoch wage ich nicht, die betreffenden Gebilde mit Sicherheit als Centrosome und Attraktionssphären anzusprechen, da einmal das Protoplasma verschiedentlich Strukturen zeigt, die Ähnliches erkennen lassen, und andererseits Fremdkörper, sowie kleine an der Zelle haften gebliebene und vielleicht tiefer in ihr Protoplasma eingedrückte Dotterpartikel zu Tiuschungen nach dieser Richtung Veranlassung geben könnten. Ich muss also die Frage nach dem Vorhandensein von Centrosomen bei den hier geschilderten Vorgängen in Folge der Ungunst des Objektes unbeantwortet lassen, obwohl es mir bewusst ist, dass ihre Entscheidung gerade bei diesen Objekten, welche die amitotische Kerntheilung so regelmäßig zeigen, von Interesse gewesen wäre.

Nachdem ich die Verhältnisse von Zelle und Kern im Allgemeinen erledigt habe, bleibt mir noch die Schilderung eines letzten Hauptbestandtheils der Zelle, nämlich des Kernkörpers ührig.

Die Größe der Kernkörper bewegt sich innerhalb weiter Grenzen, wie die Figuren der Tafel XIX zeigen. Gewöhnlich stehen sie in Korrelation zum zugehörigen Kern.

lhre Form weist reiche Mannigfaltigkeit auf. So können sie rundlich (Fig. 5 a und $b$ ), oval (Fig. $11 a, 15 a$ ), dreieckig (Fig. $6(1,14 . b)$, viereckig (Fig. 17 b), stabförmig (Fig. 10 ('), abgestumpft- (Fig. . a $a$ ) und spitzkegelig (Fig. 15 b), sichelförmig (Fig. 7 a), gerade (Fig. 10 e), oder gebogen $(11$ ( $)$, mitunter retortenartig stärker gekrümmt sein $(14(l)$. Ihre Enden sind, wie schon durch die gegebene Charakteristik theilweise bedingt, rundlich, mehr oder minder schroff abgestutzt oder in eine Spitze ausgezogen. Die mehr in die Länge gestreckten Kernkörper verschmälern sich streckenweise und schwellen dann wieder stäker an. Dies kann sich an einem und demselben Kernkürper mehrfach wiederholen.

Die Zahlenverhältnisse der Nucleolen gestalten sich nun derart, 
dass viele Kerne nur einen Kernkörper aufweisen, andere jedoch besitzen deren zwei (Fig. $3 a, 6 c, 14 b$ und c). Das verbailtnismaißig häufige Vorkommen des letzten Befundes lässt die Vermuthung rege werden, dass es sich in diesen Fällen um eine Theilung des ursprünglichen Nucleolus handeln kömle. Außerden kommen nun Formen von Kernkïrpern vor, welche das Vorhandensein zweier Kernkörper in einem Kern verständlich machen. So zeigt zunächst die Zelle $i$ in Fig. 14 einen Kernkörper mit rings um denselben verlaufender Einschnürung, die übrigens auch nur von einer Seite bemerkbar sein kann.

Das gleichzeitige Auftreten zweier Linkerbungen auf gegenüberliegenden Punkten versinnlicht Fig. $4 c$. Die nahezu bewirkte Vollendung dieses Processes ist in Fig. 5 f zu sehen. Der Kernkörper hat in diesem Zustand die ausgeprägte Form einer Ilantel. Die geschilderten Formen des Nucleolus machen das Vorkommen eines Durchschnürungsprocesses beim Kernkörper höchst wahrscheinlich.

Entsprechend der Theilung liegen nun die beiden entstandenen Tochternucleoli zunächst noch dicht an einander Fig. $3 a, 5 c$. Bald jedoch tritt ein kleiner Zwischenraum zwischen beiden auf (Fig. 14. b und 15 a). Dieser vergrößert sich allmählich. In dem Kern $d$ Fig. 8 liegen beide Nucleolen schon weit von einander ab.

In Bezug auf die Größe der durch die Theilung erhaltenen Stủcke muss erwähnt werden, dass dieselben von gleichem Umfange sein können oder aber in der Größe etwas, wenn auch nur wenig differiren. Aus dem Umstande, dass man ziemlich selten Theilungsstadien des Kernkörpers antrifft, vielmehr die Durchschnürung sich meistens schon in der Bildung von zwei Nucleolen vollendet hat, ist der Schluss gerechtfertigt, dass der Theilungsvorgang mit einer gewissen Schnelligkeit vor sich geht.

Es fragt sich weiter, wie die Lagerung der Kernkörper in den verschieden gestalteten ruhenden und in Theilung begriffenen Kernen ist. In den in der Einzabl in der Zelle vorhandenen kernen findet sich der einfache Nucleolus nahezu in der Mitte (Fig. $\{a, b d$ ). Die für die beiden Theilprodukte des Mutterkernkörpers in diesen Kernen in Betracht kommende Lagerung ist bereits bei Beschreilbung der Theilung selbst erwähnt.

Bei den in Theilung begriffenen Kernen sind immer zwei Nucleolen vorhanden, worauf ich spiter noch zurückkommen werde. In Kernen, die sich nach dem ersten Typus theilen, liegen die Kernkioper rechts und links ron der angedeuteten Theilungslinie (Fig. 1). 
Mitunter begrenzt einer derselben die Theilungsfurche dieser Kerne (Fig. $3 b, 14 e$ ).

In den Hufeisenkernen liegt je ein Kernkörper in einem der beiden Sehenkel (Fig. 6 b).

In den biskuitförmigen Kernen beherbergt jede angedeutete $\mathrm{Ab}$ theilung des Kernes einen ungefähr in der Mitte derselben liegenden Nucleolus (Fig. 12a). In den Kernen, bei deren Theilung eine centrale Öffnung in Form eines Loches oder Spaltes auftritt, liegen die Nucleolen rechts und links davon (Fig. $16 a$ und $b$ ).

Überhaupt ist zu konstatiren, worauf ich unten noch zuruckkommen muss, dass jede Kernhälfte einen Nucleolus erhält.

Bei den in doppelter Anzahl in der Zelle vorhandenen Kernen findet eine ziemlich große Regellosigkeit bezüglich der Lagerung der Kernkörper statt. Hier kommen sie eben so häufig in der Mitte, wie auch an den Enden der Kerne vor, wie aus Tafel XIX ersichtlich ist.

Bezüglich einiger anderer Eigenschaften der Kernkörper ist noch nachzutragen, dass sie zunächst in jedem Falle den Farbstoff vorzüglich annchmen. Sie erscheinen bei allen Konservirungen stark tingirt. Besonders bei Sublimatfixirung, bei der, wie erwähnt, oft die Kerne heller, als das Protoplasma bleiben, heben sie sich vorzuiglich gegen die ersteren ab (Fig. 10 und 12).

Der Kontour ist gewöhnlich deutlich sichtbar. Sie kann glatt sein oder in Ausnahmefïllen unregelmäßige Vorsprünge und Zacken aufweisen, die entweder nur streckenweise oder im Bereiche des ganzen Randes vorhanden sind (Fig. 16 b).

Ihrem sonstigen Aussehen zufolge sind die Kernkörper mitunter homogen. Oft jedoch scheinen sie auch aus an einander gelagerten Kltumpchen zu bestehen und zeigen in Folge dessen eine Art von Körnelung.

Aus den vorstehenden Auseinandersetzungen ergiebt sich ziemlich einfach, wie der Theilungsvorgang der Kerne verliuft. Jedenfalls erfolgt zunächst eine Verdoppelung des ursprünglichen Nucleolus mittels der oben beschriebenen Durchschnürung. Dafür sprechen die Theilungsfiguren desselben und der Befund von zwei Kernkürpern in den für die Theilung vorbereiteten und den in derselben begriffenen Kernen.

Die zweite Phase besteht darin, dass die entstandenen Theilstucke des Mutternucleolus mehr aus einander rúcken. In manchen Fïllen verharren die Kerne auf diesem Stadium vielleicht einige Zeit. Damit müchte ich das verhältnismäßig häufige Vorkommen von kernen mit zwei derartig gelagerten Nucleolen erklaren. Im weiteren Verlaufe geht nun die Theilung des Kernes in der ebenfalls eingehend geschil- 
derten Weise vor sich. Wie diese auch verlaufen mag, immer erhäilt jede entstehende llialfte auch einen Kiernkörper. Das Resultat des zanzen Theilungsvorganges ist, dass der Mutterkern in zwei Theilstiicke mit je einem Nucleolus zerfallen ist. Dieses Verhalten entspricht der alten Auffassung von Rear und wurde in neuerer Zeit wieder von Jounson in der Embryonalhúlle des Skorpions und von Frenzel in der Mitteldarmdrüse des Flusskrebses beobachtet.

In Ausnahmefällen kommen Abweichungen von dem geschilderten Verlauf der Theilung vor. Es kann nämlich die Theilungsfurche an einer oder beiden Seiten am Kern früher eingesetzt haben, als sich der Nucleolus getheilt hatte. Dann aber hat derselbe eine derartige Lagerung, dass or auf der Verlängerung dieser Theilungsfurchen senkrecht steht.

Ich komme jetzt zu cinem neuen Punkte, nämlich der Frage, ob die Kerntheilung mit nachfolgender Zelltheilung verbunden sei. Bei meiner Orientirung in der einschligigen Litteratur stoße ich hiufig auf Angaben der Autoren, wonach dieses Faktum, d. h. die der Kerntheilung folgende Zelltheilung nicht oder doch nicht mit Sicherheit zu konstatiren sei. Auch ich habe die Schwierigkeit der Entscheidung bei meinem Objekte zur Genüge erfahren müssen. Desshalb kann ich nur ein ohne Weiteres stichhaltiges Argument anführen, während ich im Übrigen mich auf Wahrscheinlichkeitsbeweise beschränken muss. Lnumstößlich gesichert wird die Annahme der \%elltheilung nach vorangegangener Kerntheilung amitotischer Art nur dann sein können, wenn die Beobachtungen am lebenden Objekt vorgenommen wurden, wie dies von Flemsixg und Arxoun bei Wanderzellen gemacht werden konnte. Dieses Verfahren anzuwenden, verbietet sich bei meinem Ohjekte von selbst. Als Grund für die Schwierigkeit der Konstatirung der Zelltheilung wird von mehreren Seiten der rapide Verlauf derselben angegeben.

Aus dem Umstande, dass man häufig zwischen Zellen mit DoppelKernen Paare von solchen, die nur je einen Kern besitzen, findet, und dass das Volumen dieser zusammengenommen dem der ersteren ungefiihr entspricht, kann vielleicht geschiossen werden, dass es sich in diesen Fillen um Zelltheilung handele. Diese Vermuthung wird noch durch das ganze Lagerungsverhälmis der heiden Parrlinge bestitigt

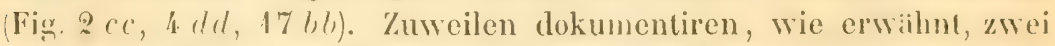
an einander gelagerte Zellen auch dureh ihre besonders starke Firhbarkeil gegenüber den umliegenden ihre Zusammengehörigheit. Diese Verhailtnisse sind an den Praiparaten selbst weit klarer und äberzeugender, als mir dies durch die Zeichmung wiederzugeben möglich war. 
Wenn Frexzer in einem Falle die Übereinanderlagerung zweier vüllig von einander abyeschnürter Zellen für einen Beweis fur Zelltheilung hält, und dieser dafür gelten kann, so sah auch ich deryleichen öfter.

Einen weiteren Stützpunkt erhält die Annahme durch den Umstand, dass zuweilen inmitten von Zellen mit zwei Kernen eine Zelle lag, welche nur einen eben so gestalteten Kern besaß, dem ganz unzweifelhaft sein Partner fehlte Fig. 10 \% Leider war es mir nicht möglich in unmittelbarer Nähe das Gegenstück dazu aufzufinden. Ich nehme desshall an, dass eine der umliegenden zweikernigen Zellen durch eine rasche Wiedertheilung entstanden ist, die bei dem erstgenannten Kern verzögert wurde.

Ein letztes, aber auch das wichtigste Argument kann jch für den Yorging der Zelltheilung noch ins Feld führen. Ofter zeigt nïnlich die Zellmembran Einschnürungen. Dicse haben in den beobachteten Fillen auf beiden Seiten zu gleicher Zeit eingesetzt. Hierdurch erhït die Zelle eine biskuit- oder hantelförmige Gestalt, den Ausdruck der bald vollendeten Theilung. Fig. $15 /$ zeigt uns dieses Verhalten in schwächerem, 76 in stärkerem Maße.

Obwohl ich nur diesem letzteren ron meinen Befunden direkte Beweiskraft, den anderen nur Wahrscheinlichkeit für den Vorgang der Zellheilung heimessen kann, bin ich in Folge des Gesammteindruckes doch zu der Ammahme geneigt, dass der Vorgang von hiufigerem Vorkommen sei, als es mir müglich ist zu erhirten. Ich möchte nochmals hervorheben, dass bezuglich der zuletzt geschilderten Verhältnisse die Präparate selbst weit überzeugender sind, als sich dies durch die Figuren wiedergeben lïsst.

Nachdem ich nun die Eigenthumlichkeiten, die das Epithel der ïteren Eificher darbietet, ahgehandelt bahe, möchte ich noch eine Schlusshemerkung daran knüpfen. Dass es sich hier wirklich um eine direkte Kerntheilung handelt, geht zunächst aus meinen thatsiichlichen Befunden hervor. Weiter fïhre ich die vollstindige $\Lambda$ bwesenheit der Mitosen an, von welchen ich hei der Betrachtung der jüngeren Eifacher zu sprechen haben werde, da sie in diesen reichlich vorkommen. Endlich möchte ich noch darauf hinweisen, diss die besondere Gröke der Kerne bei der amitotischen Theilung vielfach bemerkt und als wichtiger Punkt hervorgeholen worden ist. Dieselle ist auch im vorliegenden Falle zu konstatiren. I) Kerne des Epithels, von denen bisher gesprochen wurde, zeigen eine recht erhebliche Gröhe. Allerdings werde ich amitotische Theilung auch noch fur keme zu beschrei-

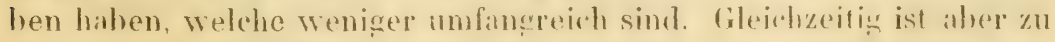


bemerken, dass in Verbintung mit diesen lefzteren auch Mitosen zu beobachten sind.

\section{B. Die jüngeren Eifächer.}

Bei den jüngeren Eifollikeln lisst sich das Epithel nicht mehr auf die erwähnte Weise mechanisch vom Dotter ablösen. In Folge dessen wurden diese Verhältnisse auf Schnitten untersucht. Für das Studium dieser jungen Stadien können nur mit Chromosmiumessigsäure konservirte Präparate Verwendung finden. Besonders gute Dienste leistet hierbei die oben angegebene starke Chromosmiumessigsäure nach der Vorschrift von Garvox. Dagegen liefert Pikrinschwefelsäure kaum, Sublimat im Allgemeinen ziemlich unbrauchbare Bilder.

Am Grunde des Keimlagers, und damit der Endkammer überhaupt liegen gewöhnlich eine Anzahl Keimbläschen inmitten der kleinen Kerne des Keimlagers. Etwas tiefer sicht man zumeist ein Keimbläschen, das schon einen ansehnlichen Dotterhof besitzt. Diese junge Eianlage füllt aber noch nicht die ganze Breite der Eiröhre aus. Weiter unten folgt dann bald eine Eianlage, bei der Letzteres der Fall ist. Diese nebst ihrem umgebenden Epithel werde ich, wie schon erwïhnt, in meinen Ausfïhrungen als das erste Eifach bezeichnen. Von nun an reiht sich Eifach an Eifach. Die Follikel sitzen zunächst mit breiter Basis an einander, so dass die im oberen Theil der Eiröhre gelegenen Fächer im Längsdurchschnitt ungefähr die Gestalt eines Parallelogramms haben. Das Verhalten der darauf folgenden Eifächer wurde bereits oben geschildert. Insbesondere wurde auf die zwischen je zwei Eifächern sich findenden Einschnürungen aufmerksam gemacht.

In der obersten Abtheilung der Eiröhre sind die Scheidewände, welche je zwei Eifächer trennen, wie auch WrLt dieses beschreibt, bald erst stückweise vollendet, bald befinden sie sich schon in voller Kontinuitat. Im ersteren Falle setzt die Bildung derselben an zwei gegenüberliegenden Punkten des Epithels ein, und die durch Zellwucherung entstandenen Zellenkomplexe vereinigen sich schließlich. In anderen Fällen geschieht die Bildung dieser Scheidewände nur von einer Seite aus.

Der Dotter erweist sich als eine unregelmiißig gekürnelte Masse, die sich durch Farbstoffe ziemlich gleichmäßig tingirt. In allen Fällen, in denen keine Schrumpfungen eingetreten sind, liegt er dem Epithel dicht an. IIier am Rande zeigt der Dotter hiiufig eine etwas andere Beschaffenheit (Fig. 18). Diese Zone besteht dann aus einem Konglomerat gröberer und kleinerer, stïrker gefïrlter Kürnchen, deren Größe und Zahl bedeutender in der Nihe des Epithels ist, und die 
gegen das Innere des Dotter's allmählich verstreichen. Dieses Verhalten ist jedenfalls auf die hier stattindende Aloscheidung von Dottersubstanz dureh das Epithel zuruickzuführen. Im Übrigen enthält der Dotter mitunter Vacuolen in Gestalt heller, deutlich kontourjerter Flecken; diese sind rundlich und von verschiedener Größe.

Wo das Keimblischen gretroften ist, erscheint es als rundliches, ovales, helles Gebilde. Seine Kontouren sind im Allgemeinen scharf gegen den Dotter abgesetzt; nur hei einem weiter unten zu erwihnenden Lagerungsverhäln is trillt dies nicht zu. Im Inneren des Keimblischens erkennt man Fiden und Körnchen, in der fü diese Kerne charakteristischen Vertheilung. Außerdem heherbergt das Keimbläschen einen bis mehrere, sehr dunkel gefiirhte Keimflecke von ebenfalls rundlicher bis ovaler Gestalt. Diese grenzen sich oft durch einen hellen Saum vom übrigen Inhalt ab und liegen meist in der peripheren Zone des Keimbläschens. Einer derselben dokumentirt sich gewöhnlich durch seine Größe als Hauptkeimfleck, wihrend die umliegenden eben so gefarbten Gebilde kleiner und verschieden umfangreich sind. Oft setzen sie sich aus einer gekörnten Masse zusammen, die von vielen hellen Stellen unterbrochen ist. Da sich der Keimfleck mit dem zunehmenden Alter vergrößert, so möchte ich ammehmen, dass die kleineren Kernkörper sich mit dem grißBeren vereingen. Die Bilder, welehe ich zu Gesicht bekam, weisen durchaus darauf hin, obwohl mir direkte Beol)achtungen dieses Vorganges nicht zu Gehote stehen.

Besonders haufig fand ich das Keimblaschen dicht an das Epithel geriickt und zwar derart, dass sich die an dasselbe grenzende Seite mehr oder weniger stark abgeplattet hatte (Fig. 18). Weiterhin zeigt sich zuweilen das Epithel gegen die Stelle hin, wo das Keimblischen liegt, breit vorgewölht und gegen diese Ausbuchtung des Epithels drangt sich dann das Keimbliischen so dicht an, dass es seinerseits eingebuchtet erscheint. Das Keimbliischen selbst sendet aber auch mitunter Fortsätze gegen das Epithel aus. Bei dieser dichten Anlagerung des Keimblischens an das Epithel wird der gegen das letatere gerichtete Kontour in manchen Fillen undeutlich, d. h. sie verschwimmt gegen die das Keimblaischen hier begrenzende Dottermasse. Zwischen Epithel und Keimblischen findet sich eine dunkle Masse vor. Diese stellt die unmittelbare Verbindung nit dem Lpithel her, dessen seharfe Begrenzung an dieser Stelle ebenfalls geschwunden ist. Dieses Verhalten wurde bereits you WVILL und Kunscuest abgehildet und niher beschrieben. Meine Befunde sind also nur eine Bestitigung der genannten Forscher, wesshalb ich nicht weiter darauf eingehe. Es handelt sich bei diesen Erscheinungen zweifellos um eine Betheiligung dos 
Keimbläschens an der emährenden Thatigheit der Eizelle. Dafur spricht ohne Weiteres die an dieser Stelle so starke sekretion von Dottermasse durch das Ejilhel, wie sie in der oben erwihnten besonders stark gefarhten Partie zum $\Lambda$ usdruck kommt. Konscuber hat ganz ahnliche Erscheinungen bei anderen Insekten und noch versehiedenen anderen Thierformen beschrieben, wobei er der auch von mir beobachteten und oben charakterisirten $\ddot{\Lambda}$ derung in Gestalt und fage des Kernes besondere Bedentung zuschreibt.

Erwihnen möchte ich an dieser Stelle noch, dass ich auch das ron Wu.t und Konscuetr beschriehene und abgebildete $\Lambda$ uftreten zweier Keimblischen in ein und demselben Ei ebenfalls einmal heobachten bonnte. Es handelte sich um eine hereits in der eigentlichen Eiröhre gelegene jüngere Eianlage.

Die aus hleinen Kernen bestehende Randbekleidung der Endkammer setzt sich unmerklich in das Epithel des ersten Eifaches fort. Das Epithel dieses vordersten Theiles der Eirühre ist im Vergleich zu den ilteren Eifichern dureh ein besonderes Verhalten ausgezeichnet. Wahrend diese letzteren, wie schon aus den friheren Darstellungen lervorging, ein einschichtiges Follikelepithel hesitzen, ist das Epithel der jüngeren und besonders der jüngsten Eificher mehrschichtiz. Die Zellen sind hier in so grofier Menge vorhanden und jedenfalls in fortwahrender Theilung begrifen, dass sie sich in dichter Mnlagerung zwisehen einander einschieben. Die Fig. 19 und 20 gehen in Theilen von Langsschnitten jüngerer Follikel ein Rild dieses Verhaltens.

Wenn sich die Eificher durch stetiges Wachsthum vergröbern, wirt die Follikelwand allmahtich diuner (Fiv. 18); die Zellen weichen nach und nach aus cinander und so kommt es, dass sehlieBlich nur noch eine einschichtige Epithellage vorhanden ist.

In den jüngeren Eifachern zeigen sich die Zellen ebenfalls deutlich ron einander aligegrenzt (Fig. 19 und 20). Besonders deutlich sind die Grenzen gegen den Totter hin. Die Epithelzellen erseheinen in diesen jingeren Eifichern bezinglich ihrer Gestaltung anders, als in den ilteren Follikeln, indem sie ziemlich stark gestreckt sind fiy. 19 und 20$)$. Die Masse dieser Zellen stell eine kontinuirliche schicht dar, die nur stellenweise dureh $\Lambda$ bselnnitte der Ermahrungsstringe unterbrouhen isl. wetche, wie erwihnt, von den einzelnen biern nach der bind- oder Nâhrkammer hinziehen.

Die kerne betinden sich theils in Ruhe, theils in Theilung. Sie sind in Lin- und Zweizahl in der Zelle vorhanden; letzores fast ehen so hioufig, wie ersteres. Ausnahmsweise fand ieh drei lierne in einer 7elle vor. 
Die Groblie der lierne rariut zwar auch, jedoch nicht in so weiten Grenzen, wie im aitteren Epithel. Zumeist ist die Hehrzahl der Kerne eines Bezirks ungefahr von gleicher Gröke. Eingestreut zwischen dieselben finden sich jedoch regrelmibig solehe, die nahezu ein doppelt so großes Volumen besitzen.

Im Gegensatz zu den Verhïlnissen im iilteren Epithel erfülen die Kerne den Inhalt der Zelle nur zum Theil und lassen so einen ziemlich groken Raum für das Protoplasma frei. Dieses letzlere erscheint etwas granulirt und firbt sich nur wenig. Die herne selbst besitzen einen mißigen Chromatingehalt und erseheinen in Folge dessen ziemlich hell. Das Chromatin ist in Form kleine liönchen unregelmäig im Kern vertheilt, der dadurch granulirt erscheint.

Die Form der Kerne ist im Allgemeinen linglich, der zugehörigen in der Regel eben so gestalteten Zelle entsprechend. Jedoch können in anderen Fillen auch der Lingen- und Querdurchmesser ungefailır pleich sein. Die Form der Kerne ist dann mehr gedrungen und rundlich (Fig. $18 \mathrm{c}$ ).

Die Kontouren der Kerne sind stets gut ausgeprigt und ghtatt. Dies gilt auch für diejenigen Kerne, welche sich uns als Theilungsstadien zu erkennen geben, wie wir sogleich noch sehen werden. Ziemlich oft sieht man Kerne, die den hisuligen Befund won zwei hernen in einer Zelle verstandlich machen. Jedoch mus man kritisch zu Werke gehen, weil man nie recht wissen ham , ol man den hern in seiner Totalitit - oder nur angeschnitten vor sich hat. Desshall, wage ich nicht zu hehaupten, dass hier analog gewissen Kermen in Epithel der ilteren Eifacher eine furche rings un den hern verlanfen lionnte. Trotzdem weisen manche kerne meiner l'riparate, fon denen man mit grofer Wahrscheinlichkeit amehmen hamn, dass sie in toto rorliegen, dieses Verhalten auf. Dagegen kann es lieinem Zweifel unterliegen - oh die lierne in ihrer Totalibit oder nur zum Theil rorliegen, ist hierbei frefol-

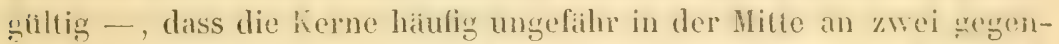
iiberliegenden Punkwn oder von cince seite her cinsetzende linschnii-

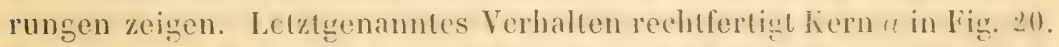

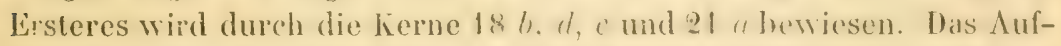
treten eines Loches oder Spaltes in thm Kern heobahtete ich im Epithel

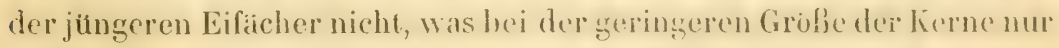
naturlich ist. In Folge des Lagerungsrerhibnisses mancher Kormparare in einer Zelle (Fig. 19 a, 20 b) ist viclloicht trotzdem der Sihluss gorechtfertigt, dass eine Durehschnitung von ohen her stattinden kann. In genamnten Fälen stoken nambleh die Pare mit geraden, den ganzen Querdurehmesser des Lernes einnehmenden Seiten an einander. 
Das Vorkommen von einem und zwei Kernen in einer Zelle einerseits und die geschilderten eingeschnürten Kerne andererseits lassen auch für die Kerne des Epithels der jüngeren Eificher die Annahme der amitotischen Kerntheilung als gesichert erseheinen. Diese Auffassung werden die sogleich mitzutheilenden Verhältnisse der Kernkörper noch besser stützen. Der Umfang der Kernkörper richtet sich im Allgemeinen nach demjenigen des Kernes. Viele Kerne beherhergen nur einen Nucleolus. Eben so häufig finden sie sich aber auch in der Zweizahl in denselben. In seltenen Fallen kommen drei Kernkirper vor (Fig. 21 a).

Ihrer Form nach weisen sie große Mannigfaltigkeit auf. Sie können rundlich, oval, ellipsoid, spindel-, strang-, wurstförmig, gerade oder sehwach gebogen sein (Fig. 18, 19, 20). Ihre Enden könuen sich nach einer oder auch nach beiden Seiten hin verdicken, in anderen Fällen mehr oder weniger spitz zulaufen. Die Kernkörper zeigen sich scharf und glattrandig begrenzt. Sie färben sich intensiv und treten dadurch in dem heller gefäbten Kernplasma deutlich hervor.

Analog dem für die liernkörper in den ailteren Eifächern geschilderten Verhalten finden auch bei den Nucleolen der jüngeren Follikel Durchschnürungen statt. Unschwer sind alle Zwischenstufen von deı nur angedeuteten bis fast vollendeten Durchschnirung aufufinden. Der Kernkörper des Kernes $b$ in Fig. 2) 1 hat eine einseitige Einschnirung erlitten. Bei den Kernkörpern der Kerne $a$ und $b$ in Fig. Is, $c$ und $f$ in Fig. 20 ist die Einschnurung beiderseits aufgetreten, wodurch eine Hantelform resultirt. Durch die Vollendung des Durchschniurungsprocesses entstehen dann schließlich zwei Kernkörper, die zunächst noch dicht an einander liegen (Fig. 20 g), bald aber durch einen Abstand getrennt gefunden werden.

Die seltenen Fäle, in denen drei Kernkörper (Fig. 21 a) vorhanden sind, erklairen sich leicht in der Weise, dass sich der eine der entstandenen Tochternucleoli rasch weiter getheilt hat. Es bratucht nach dem Vorausgegangenen kaum hemerkt zu werden, dass die zuletzt geschilderten Verhältnisse der Kernkörper ehenfalls mit dem Theilungsvorgang der Kerne in Verbindung stehen.

Derganze Theilungsprocess geht somit auch im Eipithel der jungeren Eifächer in der für die älteren Follikel geschilderten Weise vor sich, so dass ich so eingehend, wie fruher, nicht dariber zu handeln brauche. Als eine Besonderheit habe ich nur das Vorkommen von zwei liernkörpern in einer noch nicht ganz algegliederten Hialfte der lierne zu erkliren (Fig. 21 "). Dieses Verhalten scheint mir fiir eine rasche Weitertheilung der Tochterkeme zu sprechen, in :ihnlicher Weise, wie ich dies 
auch für die Kerne der äteren Eifacher wahrscheinlich zu mathen suchte, und wie sich dies auch weiterhin bei den fiemen des keimlagers nochmals wiederholen wird.

Neben der Theilung nach dem Typus der Amiloseund, fast mitgleicher Haufigkeit, kommen auch Mitosen vor, wenigstens verhät sich dies in den jüngsten Eifächern in dieser $\mathbf{W}$ eise. Da ich bei meinen Untersuchungen von den äteren Eifachern ausging, deren Epithel auf die angegebene Weise an Ol,erflächenbildern studirt wurde, so entgingen mir zunächst die mitotischen Figuren, doch fand ich sie dann bald und reichlich auf Schnitten durch gut konservirte jüngere und jüngste Eifächer. Bezijglich des Vorkommens von Mitosen bieten die untersuchten Eiröhren gewisse Verschiedenheiten dar. In den einen finden sich nämlich Phasen der indirekten Theilung in großer Anzahl, während sie in anderen, wenn auch nicht ganz fehlen, so doch nur spärlich vertreten sind. In Folge dessen möchte man daran denken, dass eine gewisse Periodicitai in Auftreten der Mitosen besteht, die veranlasst, dass sie sich das eine Mal besonders hiiufen, wihrend das andere Hal nur wenige vorhanden sind.

In der epithelialen Schicht sind die mitotischen Figuren leicht dureh ihre Größe und die Anordnung der tief dunkel gefirloten Chromosome zu erkennen. Sie befinden sich in den verschiedenen Stadien der indirekten Kerntheilung. Besonder's häufig sind Spindeln nit Ärquatorial- oder Tochterplatten. Die Keruspindeln zeichnen sich gegenüber den umliegenden liernen durch ihre Grölbe aus (Fiğ. I!) u. 20). Zumal sind sie breiter als jene. Die Fasern der Spindel sind mehr oder minder deutlich sichtbar.

Ihrer Lage nach werden die Mitosen an den versehiedensten Stellen des Epithels der jüngeren Eifïcher gefunden. Mit einer gewissen Hiiufigkeit jedoch kommen sie an den Übergangsstellen vom Epithel in die Seheidewinde, welche die einzelnen Eificher trenuen, vor. Die Erklarung fur dieses Verhalten ist in der Amnahme zu finden, dass in diesen Partien die Zellthcilung eine besonders rege ist. Zumeist finden sich die Mitosen vereinzelt; zuweilen sieht man zwei von ihnen neben einander liegen, mitunter treten sie in Nestern zu funf bis sechs auf, dann allerdings durch einige andere Kerme getrennt.

Die Mitosen sind, wie sehon kurz erwihnt, auf keinen bestimmten Bezirk beschränkı, sondern sie kommen ganz gewöhnlich in unmittelbarer Nahe amitotisch sich theilender Kerne vor. Neben den Mitosen könnte ich die verschiedensten Stadien der amitotischen Theilung finden. Z. B. sind in den Fig. 19 und 20 außer den Mitosen ami- 
tolische Kerntheilungsstadien auf verschiedenen Ausbildungsatufen bemerkbar.

Wir stoBen hier zum ersten Nale auf ein Verhalten, wie wir es weiter unten noch verschiedentlich zu konstatiren haben werden, wonach beide Arten der Theilung an ein und derselben stelle neben einander hergehen können. Da man in den äleren Eifachern nur Amitosen findet, so handelt es sich nummehr um die Entscheidung der Frage, wo das Nebeneinandervorkommen beider Typen aufhïrt. Durch genaues Zählen der Eificher und Vergleichen vieler Eirohren konnte ich feststellen, dass die Mitosen nicht weiter, als his zum neunten Eifach hinunterreichen, wobei in der schon früher aus einander gesetzten Weise dasjenige Ei, welches als jüngstes die ganze Breite der Eiröhre ausfullt, als Nr. 1 bezeichnet wird.

In der Regel reichen die Mitosen allerdings nicht so weit in der Eiröhre nach hinten, sondern sie werden fúr gewännlich nur bis zum seohsten Eifach gefunden. Immerhin sind die Fille nicht selten, in denen sich die Mitosen ubber das sechste Eifach hinaus weiter nach linten erstrecken. Im neunten Eifach sind nur verhailnismaßigy selten noch Mitosen vorhanden.

In Riülisicht auf die Iliufigkeit des Auftretens der Mitosen in diesem Jesehrinkten Bezirke der Eiröhre muss noch erwihnt werden, dasi dieselhen gewohnlich in den jügsten bifichern an zahlreichsten sorhanden sind, in den daransehliekenden aber allmihlich ahnehmen. Immenh hann aber auch das letule Fifach, das sic gerade heherbergt, auf eincm selhnitt noch deren zwei oder gar drei aufweisen. Im neunton bifach allerdings sind sie, wie sehon erwihnt wurde, seltener.

Die fü die Zellen, Kerne und Kernkörper dargethanen Verhailnisse bestehen in Allgemeinen auch fü den vorderen Theil der Eirohre ohne Weiteres zu Rerht. Abweichungen haben nur in der Weise statt, dass die Kerne nach und nach an Gribe zunehuren, die Mitosen, wie erwibut, allmahlich seltener weren und schließlich ganz verschwinden. Bald alore, oder genauer gyesagt, rom achten oder neunten Eifach an, fritt damn in so fern eine Differenzirung im Epithel auf, als in dem nach rom am Follikel gelegenen und oben besehriehenen Aufsatz dic Zellen und kerne eine auffalbende Gröbe zeiggen Fig. 18). Besonders

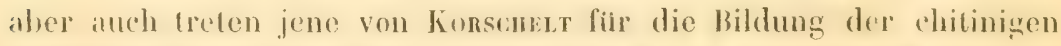
Slrahlen der alteren Eier in Anspruch genommenen merkwirdigen und auberst umfangreichen Zellenpare am Grunde des $\Lambda$ ufsatzes auf.

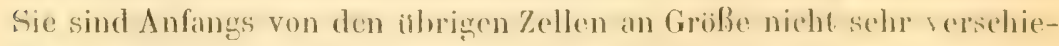
don, Wachsen aber hald selw hedeutend. Zwei der Zellen vereinigen sich zu den Doppelzollen, innerhalb deren dann erst spiter die Bildung 


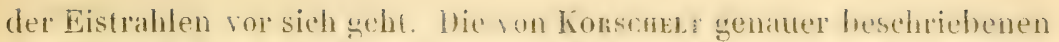
Verhältnisse habe ich so weit verfolgt, dass ich die wesentlichen Punkte dieses eigenartigen Vorganges dur Chitinbildung hestitigen kann. Erwihnen möchte ich besonders als das auffaillieste Verhalten dieser Zellen, dass ihre Ken ne die von Konsunet als Oberflachenvergrißerung zur Betheiligung an der \%ellthitigkeit gedeuteten Fortsitze aufweisen. Diese Oherflachenvergrößerung findet an den gegen einander gerichteten Rindern der herne statt, wie die Fig. 18 / u. dies erkemen liisst. Die hier gezeichneten, noch ziemlich jungen lierne der Doppelzellen crscheinen bei der Konservirung mit Chromosmiumessigsiture und Färbung mit Ilimatoxylin heller, als das Zellplasma, was allerdings besonders auch daher kommt, dass sich das Plasma zwisehen beiden Keruen, d. h. an der Stelle, wo spater die Strahlenbildung stattfindet. auffallend stark färht, wie die Fig. 18 dies ebenfalls zeigt.

Außer den sicben großen Zellparen (Doppelzellen) enthiilt der Aufsatz der Eificher eine Menge von Epithelzellen, welehe die ührigen Zellen des Follikels an Größe ziemlich bedeutend übertreffen. Wie aus der Fig. is hervoryeht, sind dieselben zumal am vorderen Theil des Aufsatzes stark in die Lainge gestreckt. Diese Zellen zeigen Verhält-

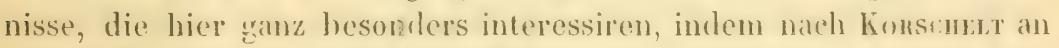
dieser Stelle die amitotischen Theilungen besonders zahlreich vorkommen und zu einer starken Vermehrung dew'zellen führen sollen, welche mit der weiteren Ausgestaltung des Aufsatzes und der Verlagerung der Doppelzellen in Verbindung steht. In den ron mir heohachteten Stadien der Aushildung des Aulsatzes fand ich chenfalls Amitosen ror.

Eines eigenthimblichen, eimmaligen Befundes moghte idh an dieser.

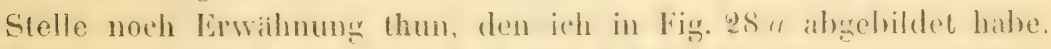
Dieses merkwärdige Gehilde fand sich auf der Scheidewand zwischen dem funften und sechsten kifach einer biröhe unweit der Vophindungsstelle ron Eppitiel und scheidewand. Es hatte an diesere stelle die letztere ausgebuehtet; wihrend sich die Wand im weiteren Verlanfe wieder verschmailerte. Das eine Inrittel derselhen hatte sich dunkel gefärbt, während der Rest heller geblieben war.

Das fragliche debilde war von ovaler festalt und wies einen

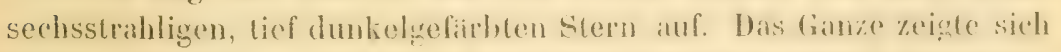
ron einem hellen und dentich kontourirtens saum ungehen, der jedenfalls in Folge von Schrumpfung entstanden war.

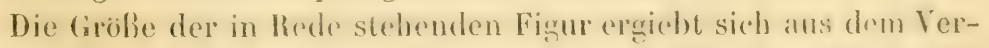

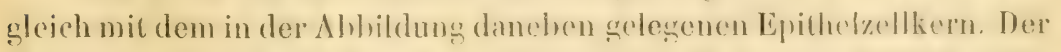
Kontour war undeutlich und rersehwommen. Mas gature Gehilde hatte sich sehwach gefärht. Seinem sonstiggen Aussehen zufolgूe erschiom m. 
homogen bis auf die jetzt näher zu beschreibende Differenzirung. Diese bestand aus sechs Reihen tief dunkel gefirbter an einander gelagerter Kürnchen. In Folge des in der Mitte stattfindenden Zusammenstoßens dieser Linien erlangte das Ganze das Ansehen eines Sternes. Die Körnchen, welche die Reihen bildeten, ließen zumeist kleine belle Zwischenrïume zwischen sich und waren so gelagert, dass eine leicht geschlängelte Linie entstand. Theilweise fanden sich die körner auch neben einander vor.

Ähnliches bcobachtete ich auch bei Notonecta. Ich fand bei dieser Form einige Male Figuren, wie sie in Fig. $28 b$ und $c$ abgebildet sind. Diese Gebilde bestehen aus einer mehr homogenen Zone, in der an ciner Stelle ein heller Fleck, vielleicht eines der Centrosome, sich befindet, und den in Form eines Dreistrahls angeordneten, tief dunkel gefirbten Chromosomen. Diese haben sich zu drei Reihen an einander gelagert, welehe in der Mitte zusammenstoßen. Es kann kein Zweifel sein, dass es sich um abnorme Kerntheilungsfiguren handelt, wie sie wiederholt von anderen Autoren beschrieben worden sind. Ähnliche Gebilde wurden von Connal, Denss, Haxsemann in bösartigen Geschwillsten, wie Carcinomen und Sarkomen, von Schotrlïnder im artificiell entzundeten Auge, von Fol und Ilentwig bei der Polyspermie beobachtet. Dessgleichen hat A. Brater derartige abnorme karyokinetische Figuren im Ei von Artemia beschrieben, und Hesse theilt neuerdings Entsprechendes äber den Hoden von Oligochaeten mit.

\section{Das Keimlager.}

Das Keimlager bildet, wie früher bereits erwahnt wurde, den hinteren Abschnitt der Endkanmer. Nach vorn zu stößt es an den Nährzellenkomplex der letzteren; auf der anderen Seite wird es von den jüngsten Eifichern begrenzt. Für das Studium des Keimlagers erweisen sich hauptsichlich die durch die mittleren Partien der Eiröhre geführten Längsschnitte günstig.

Das Keimlager besteht aus einem Konglomerat kleinster, nur bei Beobachtung mit stirkerer Vergrößerung deutlich unterscheidbarer Kerne, die zumeist in eine gemeinsame l'lasmamasse regellos eingebettet zu sein scheinen (Fig. 23). Nur selten lassen sich die Zellgrenzen deutlich machen. Dureh die Ernährungsstränge wird der Zellenhaufen gewöhnlich in zwei oder mehrere, parallel verlaufende Felder zerlegt. In Fig. $26 a$ ist ein Theil eines solchen Ernährungsstranges abgebildet. In der Masse der kleinen Kerne seitlich, wie auch in der Mitte gelegen, finden sich einige Keimbläschen in jungen Eianlagen vor. Sie sind durch ihre Größe und hellere lärbung kenntlich. Gelegentlich tritt 
eine oder die andere Nihrzelle weiter unten auf, wihrend im Allgemeinen die Grenze gegen den Bezirk der Nihrzellen scharf abgesetzt ist.

Gegenüber den Kernen des Epithels zeigen die des Keimlagers einen etwas größeren Gehalt an chromatischer Substanz. Fast stets sind neben dem bezw. den Kiernkörpern mehrere stark gefürbte Ghromatinbrocken zu unterscheiden, welche den Kernen ein grob granulirtes Aussehen verleihen.

Die Kerne sind von nicht sehr verschiedener Größe (Fig. 22-26). Ihre Form ist aber recht different. Im Allgemeinen sind sie rundlich oder oval. Es kommen aber auch in anderen Fällen mehr in die Liinge gestreckte Kerne vor (Fig. $23 d$ ).

Auch im Keimlager befinden sich die Kerne sowohl in Ruhe als auch in Theilung. Da die Zellgrenzen fehlen oder in seltenen Fällen nur sehr undeutlich zu erkennen sind, kann hier von Zellen mit zwei Kernen nicht gesprochen werden. In Folge dessen kann ich mich in der Annahme von der Theilung amitotischer Art der Kerne des Keimlagers nur auf besonders geformte Kerne stützen, welche für diese Art der Theilung sprechen. Die Kerne $a$ in Fig. 24 und $b$ in Fig. 26 zeigen Stadien der direkten Theilung, indem in der Mitte eine Einschnürung aufgetreten ist. Kern $a$ in Fig. 22 und a in Fig. 2:) illustriren diesen Process in weiter fortgeschrittenem Zustand. Ein eigenthümliches Vorkommnis möchte ich an dieser Stelle noch erwähnen. In manchen Fällen sticht nämlich der in Theilung begriffene Kern in Folge der stärkeren Fürbung von den umliegenden Kernen auf den ersten Blick ab. In Fig. 23 a habe ich dieses Verhalten ab)gebildet.

Die Kernkörper können die verschiedensten Formen besitzen, wie die Fig. 22-26 zeigen. 'Stets nehmen die Nucleolen den Farbstoff vorzůglich an.

Viele Kerne besitzen nur einen Nucleolus. In anderen Fiallen sind zwei vorhanden (Fig. 23c, 26 l). Analog den an anderer Stelle mitgetheilten Befunden sind auch im Keimlager Durchschnürungsstadien von Kernkörpern aufzufinden (Fig. $23 b$ und $d)$. In Fig. 24 ( sieht man die Hälften kurz nach dem Zerreißen der Verbindungsbrücke, die an einem Theilstucke sitzen geblieben ist. Einige wenige Male wurden auch, wie schon oben für das Epithel erwithnt, in der einen Miilfte des in Theilung begriffenen Kernes im Keimlager schon wieder zwei Kernkürper wahrgenommen (Fig. 2!) (1). Dieses Verhalten spreche ich, wie auch schon früher, als einen Beweis für die angebahnte rasche Wiedertheilung des zukunftigen Theilstückes des Mutterkernes an. 
Auch in den Kernen des Keimlagers kam also der gesamme Theilungsprocess nach dem Typus der Amitose verlaufen.

Auf Grund der Durehsicht einer großen Anzahl ron Priparalen halte ich mich zu der Anmahme berechtigt, dass der in der geschilderten Weise vor sich gehende amitotische Theilungsvorgang im Keimlager dic Regel ist.

Andererseits jedoch konute ich auch zuweilen Mitosen inmitten der kleinen Kerne des Keimlagers konstatiren, doch muss ich ausdrücklich hervorheben. dass dies nur selten der Fall war, zu meiner Überaschung, möchte ich sagen, denn ich hätte geglaubt, gerade in diesem Theil der Eiröhre die Mitosen besonders häufig zu finden.

Im Keimlager stieß ich, wie sich aus dem Vorstehenden ergiebt, zum zweiten Mal auf die interessante Thatsache, dass heide Theilungsarten neben einander hergehen können. Ein Unterschicd gegen das gleiche Verhalten im Epithel der jüngsten Eifacher liegt nur in der verschiedenen Ilïufigkeit des Vorkommens, da in den jüngsten Follikeln die Mitosen den in direkter Theilung begriffenen Kernen ungefihr das Gleichgewicht halten können, während sie im Keimlager, wie erwähnt, nur selten auftreten.

\section{Der entleerte Follikel.}

Der entlecte Follikel sitzt, wie bereits erwibnt, als $\Lambda$ fangstheil des Eileitersticles diesem kappenförmig auf und geht nach hinten zu in denselben ublor.

Nachdem das vom Ei verlasseue Fach seine Funktion erledigt ha!, ist es dem Untergange und der Resorption geweiht. Letztore selueint bald nach dem Entlassen des Eies vor sich zu gehen und ziemlich rasch um sich zu greifen. Keiner der untersuchten entleerten Follikel wies vollkommen intakte Verhätnisse auf, wenngleich die Eificher attch in Riicksicht auf die in geringerem oder starkerem Grale vorgeschrittenere Degeneration mannigfache Verschiedenheiten zeigen. In Folge dessen kamn man stets zwischen mehr oder minder normalen El'menten, in Zerfall begriffenen und zu einer unförmigen Masse geworkenen Zellen unterseheiden. Man hezeichnet dieses Gehilde belimntlich mit einem den Verhailuissen der Wirbelthere entehnten Namen als Gorpus luteum. Ich fand dasselbe entsprechend der Zeildauer, seit woldher der Follikel vom lï verlassen war, in recht versehiedener Beschallenheit vor.

Das letzte fifach und der entleerte Folliked sind zumcist, wie auch oben schon angegeben wurde, in Folge Umsehlagens des Verbindumgsstitckes dicht an cinander gerilckt. Die noch nicht in Auflisung be-

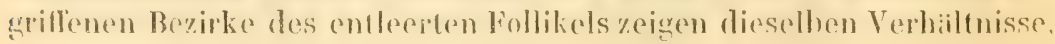


wie sie im letzten Eifach vorhanden sind. Kern und Kernkïrper differenziren sich in ganz der nämlichen Weise. Nur die Zellgrenzen sind undeutlich geworden. Es finden sich zumeist Zellen mit zwei Kernen. Seltener kommen einkernige Zellen vor. Ich fand sogar noch solche, deren Kerne noch in (amitotischer) Theilung begriffen sind.

Auch die Eigenschaften der Kernkörper stimmen, wie das ja auch nicht anders zu erwarten ist, mit den oben geschilderten Verhiiltnissen uberein.

Dieses normale Verhalten bleibt am längsten auf der Kuppe des entleerten Follikels bestehen, während der Resorptionsprocess an den Seitentheilen schnellere Fortschritte macht. Derselbe geht in der Weise vor sich, dass zunächst der Kernkörper seine Differenzirungsfähigkeit durch Farbstoffe verliert. Er tritt allmählich ganz zurück. In Folge dessen erscheint der Kern als ein ziemlich gleichmäßig tingirtes und gekörneltes Gebilde. Iland in Hand mit diesen Verainderungen geht eine Verkleinerung der in Rede stehenden Elemente. Noch weiter in der Degeneration fortgeschrittenere Kerne haben mehr und mehr an der Aufnahmefähigkeit von Farbstoffen eingebüßt, und ihre Körnelung verliert sich. Der Inhalt des Kernes wird fast homogen. Zuweilen sind die Umrisse der früheren Gestalt der Kerne noch leidlich zu erkennen, oft sind die Kerne jedoch schon zu unfürmigen Massen geworden. Das Resultat dieses Zerstörungsprocesses ist ein Konglomerat von in verschiedenem Grade gefäbten und farblosen Schollen und Brocken, die regellos an einander liegen. Weiter bin ich dem Auflösungsprocess der Follikel nicht nachgegangen, da er für die von mir verfolgten Zwecke keine Besonderheiten mehr bietet.

Die Degenerationsprocesse verlaufen, so weit sie die Seitentheile betreffen, unter dem gleichen Bilde, wie es soeben für die Kuppe des entleerten Follikels geschildert wurde, nur seizen sie hier friher ein, als an letzterer. Es scheint, als ob die Seitentheile nicht völlig zerstiort würden, sondern zum Theil direkt in den Eirührenstiel übergingen. Diese Verhältnisse sind bereits von Konscriezt studirt worden, doch haben mich meine ausgedehnteren Untersuchungen derselhen etwas weiter geführt, so dass ich in Kur\%em darauf eingehen möchte.

Durch den Austritt des Eies wird die hintere Wand des Follikels und die Kuppe des Eiröhrenstieles durchbrochen, so diss dinn die crhalten gebliebene vordere Wölbung des entleerten Eifaches cine Zeit lang den Abschluss des Ovarialtractus nach außen bildel. Wie meine Präparate zeigen, entsteht darauf eine Decke des Eirührensticles von Neuem. Dieses geschieht in der Weise, dass von den unversehrt ge-

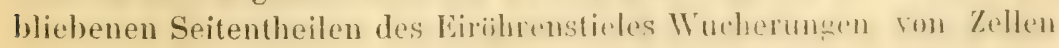


ausgehen, die einander entgegenwachsen. Das Resultat ist, dass je wehr der entleerte Follikel durch Resorption schwindet, die neugebildete Decke des Eiröhrenstieles an Mähtigkeit zunimmt und schließlich eine, wenn auch geschlossene Verbindung mit dem nun zum letzten Follikel gewordenen Eifach herstellt.

Endlich sei noch bemerkt, dass das Corpus luteum des entleerten Follikels bei diesem Neubildungsprocesse der Decke des Eiröhrenstieles in das Innere dieses letzteren verlegt wird. Bei den beschriebenen Vorgängen der Wiederausbildung der vorber zerstorten Epithelien fand ich niemals Mitosen, obwohl ich solche hicr zu finden erwartet hätte. Die Theilung der Kerne erfolgt stets auf amitotischem Wege, so weit ich dies beobachtete.

E. Das Epithel des Eiröhrenstieles.

Der Eiröhrenstiel stellt, wie oben bereits erwähnt wurde, ein schlauchförmiges Gebilde dar, das mit vielen Falten und Krausen versehen ist. Vorn sitzt ihm der entleerte Follikel auf, hinten beherbergt er gewöhnlich ei ausgestoßenes Ei.

In Folge der faltigen Beschaffenheit erscheinen die Zellen auf einigen Punkten polygonal (Fig. 27), auf anderen mehr länglich und mit den Breitseiten an einander stoßend, Dazwischen finden sich Übergänge.

Die Zellgrenzen sind im Allgemeinen deutlich. Die an das Lumen des Eiröhrenstieles stoßenden springen in dasselhe in schwachem Bogen vor, so dass der Rand einer derartigen Partie eine gewellte Linie darstellt.

Die Kerne des Eiröhrenstieles zeichnen sich besonders durch ihren Reichthum an Chromatin aus (Fig. 27). Dasselbe ist in versehieden großen, stark gefärbten Brocken regellos in den Kernen vertheilt. Kerne und Zellen sind im Eiröhrenstiel kleiner, als die der eigentlichen Eiröhre.

Die Kerne sind ferner zumeist inmitten der Zelle gelagert und lassen eine größere periphere Zone für das Protoplasma frei. Dieses färbt sich in der vorderen Partie des Eiröhrenstieles ziemlich stark, während es in dem hinteren Theile den Farbstoff nur wenig annimmt.

Auch im Eirührenstiel sieht man zumeist zwei Kerne in einer Zelle liegen. Seltener kommen solche in Einzahl oder Theilungsstadien vor. Die Theilung vollzieht sich auch hier nach dem Typus der Amitose. Für dieselbe gilt das hei frihteren Gelegenheiten Gesagte mil Ausnahme der Kernkürperverhailtnisse. Desshalh verweise ich, 
um mich nicht unnüthig wiederholen zu müssen, auf obige Schilderung und werde nur auf die Abweichungen eingehen.

Wenngleich sich zuweilen ein Chromatinbrocken durch seine Größe vor anderen als Kernkörper auszeichnet (Fig. $2 \% b$ und c), ist die Größendifferenz von den anderen Ghromatinkürnern gewöhnlich doch nur gering.

Dass es sich wirklich im Eiröhrenstiel um amitotische Kerntheilungen handelt, wird zunächst durch das häufige Vorkommen von zweikernigen Zellen wahrscheinlich gemacht. Zu größerer Sicherheit gelangt diese Annahme durch die thatsächlichen Befunde der in Durchschnurung begriffenen Kerne (Fig. 27 a und $c$ ). Weiterhin wird sie durch die vollstindige $A b w e s e n h e i t$ von mitotischen Figuren unterstutat. Nach der Theilung bleiben die entstandenen Stücke gewöhnlich nahe an einander gelagert, wenngleich sie auch in seltenen Fällen weiter von einander getrennt gefunden werden (Fig. 27 d). Es fragt sich jetzt, wie sich das Chromatin bei diesem Theilungsprocess verbailt. Das ziemlich seltene Vorkommen eines Kernkörpers in Kern wurde bereits erwähnt. Nie konnte ich zwei wirkliche Nucleolen, wie bei den früher geschilderten Kernen in den Kernen des Eiröhrenstieles nachweisen. In Folge dessen kommt bei der Theilung ohne Weiteres eine gewisse Anzahl Chromatinbrocken auf jede Hälfte des Kernes. Wo ein Kernkörper vorhanden ist, muss ich annehmen, dass derselbe vor der Theilung des Kernes in mehrere Stücke zerfillt und sich in Folge dessen nicht mehr vor den übrigen Chromatinbrocken auszeichnet.

An der Stelle, wo das mit dickem Chorion versebene, ausgestoßene Ei liegt - und dieses ist immer am Ende des Eiröhrenstieles der Fall, - hat eine gewaltige Ausdehnung des letzteren stattinden müssen. Diese wird durch die Muskulatur und die Faltungen der Wand des Eiröhrenstieles ermöglicht. In Folge dieser Ausdebnung sind die Zellgrenzen undeutlicher geworden. Die Kerne liegen hier weiter von einander entfernt, lassen aber dieselben Verhältnisse erkennen, die soeben geschildert wurden.

\section{F. Die bindegewebige Hulle.}

Die Struktur der bindegewebigen Ilülle und des Endfadens wurde bereits von WiL. und Konscher.T eingehend beschrieben. Desshalb beschrïke ich mich, abgesehen von einigen nothwendigen Erklirungen, im Übrigen ausschließlich auf die Kerne und deren Theilungsverhältnisse.

Die bindegewebige llülle liegt der Eiröhre als ïußere Bekleidung ziemlich dicht an. Sie baut sich aus Zellen auf, deren Grenzen mehr 
oder minder dentlich sichtbar sind. Die Zellen sind von rundlicher, ovaler oder in anderen Fillen von polygonaler Form.

Die Zellen enthatten in der Regel einen einzigen Kern, der sie nur zum Theil ausfült. Es bleibt in Folge dessen ein verhïltnismaíig breiter protoplasmatischer landsaum, der ziemlich homogen erscheint. In seltenen Fällen liegen zwei Kerne in einer Zelle.

Die Größe der Kerne variirt. Im Ganzen sind sie bedeutend kleiner, als die früher besprochenen kerne, d. h. die der eigentlichen Eiröhre.

Ihrer Form nach können sie rundlich oder oval, in anderen Fällen jedoch mehr länglich sein.

Ihrem sonstigen Aussehen zufolge tragen die Kerne der bindegewebigen Hülle einen anderen Charakter, als die nebenliegenden Kerne der Eiröhre zur Schau. Auf die Unmöglichkeit einer Verwechselung mit den Kernen der letzteren macht auch schon KonscneLt aufmerksam. Sie besitzen nämlich einerseits einen mäßigen Gehalt an Chromatin, das in kleinen Brocken regellos im Kern zerstreut ist, andererseits liegen sie ziemlich weit aus einander.

Withrend meistens ein eigentlicher Nucleolus fehlt, so tritt doch zuweilen einer der Chromatinbrocken an Größe mehr hervor, so dass man ihn wohl als Kiernkörper ansprechen könnte.

Das häufige Vorkommen von Zellen mit einem Kiern deutet von vorn herein darauf hin, dass in der bindegewebigen Hülle im Gegensatz zu den bisher abgehandelten Kernarten kein reger Theilungsvorgang obwaltet. Dieses Verhalten ist auch in Rúcksicht auf die Funktion der bindegewebigen Hülle ganz verständlich. In der That sind dann auch solche Formen von Kernen, die auf Theilung schließen lassen, selten. Der erwähnte Befund von einigen zweikernigen Zellen legt es nahe, dass es sich auch in der bindegewebigen Hulle um Amitose handelt. Weiterhin zeigen sich dann auch zuweilen Phasen der direkten Kerntheilung, indem in der oben geschilderten Weise Einschnurungen aufgetreten sind. Jedoch mache ich nochmals darauf aufmerksam, dass dieses Verhalten selten ist.

Wie sich das Chromatin bei diesem Theilungsprocess verhïlt, ist schwer zu entscheiden. Fur die Mehrzahl der Fälle muss ich eine Vertheilung einer gewissen Anzahl Brocken auf beide in Bildung begriffenen IIalften des Kernes annehmen. Andererseits kann sich in jedem angedeuteten Theilstück des Kernes ein größeres Chromatinkorn befinden, das einen Kernkürper vorstellt. Dann müsste man naturlich an eine Theilung des letzteren denken.

Außer den besprochenen Theilungsfiguren fand ich in der binde- 
gewebigen Hulle auch einige wenige Mitosen auf. Bei der Durchsicht einer großen Anzahl von Schnitten sah ich drei Mal solche. Eine davon traf ich im Bezirke der Endkanmer, zwei in dem der eigentlichen Eiröhre an.

Meinen Gesammteindruck von den Theilungsverhailtnissen in der bindegewebigen Hülle der Eirühre fasse ich nochmals, wie folgt, zusammen: Gegenüber den bisher beschriebenen kernarten sind Theilungsvorg:inge im Allgemeinen selten. Die Theilung geht noch am häufigsten auf amitotischem Wege vor sich, jedoch kommt, wenn auch allem Anschein nach recht selten, mitotische Theilung vor.

\section{G. Der Endfaden.}

Auch in Bezug auf den Endfaden verweise ich zur Orientirung theils auf die Abbildungen und Angaben von Konschelt und Wilf, theils auf meine oben gemachten Ausführungen.

WiLl sagt, dass die Kerne des Endfadens in eine gemeinsame Plasmanasse eingebettet sind. Wenngleich ich num auch zumeist den gleichen Eindruck erhielt, so sah ich in anderen Fillen Andeutungen von Zellgrenzen. Jedoch zu einem klaren Bilde dieser Verhältnisse gelangte auch ich nicht.

Die Kerne, welche erst bei stärkerer Vergrößerung deutlich sichtbar sind, weisen eine verschiedene Größe auf.

Zumeist besitzen sie einen tief gefärbten Nucleolus von differenter Gestalt. Daneben kommen noch Chromatintheile in Form ron Brocken vor. Zuweilen jedoch finden sich zwei Nucleolen. Dieses Verhalten erinnert an die oben beschriebenen Beobachtungen. Des Weiteren finden sich auch in Zerschnürung begriffene Kernkürper.

Auch die Theilungen des Kernes gehen in der früher besprochenen (amitotischen) Weise vor sich. Sie sind nicht gerade allzu hïufig, jedenfalls jedoch übertreffen sie die in der bindegewebigen Hülle an Zahl.

Durch meine früheren Befunde, welche beide Arten der Kerntheilung neben einander hergehend zeigten, vermuthete ich $̈$ hnliches auch im Endfaden zu finden und suchte mit Aufmerksamkeit nach Mitosen. Jedoch bei der Durchsicht einer großen Anzahl von Schnitten war es mir nicht möglich, Phasen der indirekien Theilung zu konstatiren. Auch bei Eiröhren, deren Epithel in reichlicher Menge Mitosen aufweist, sind letztere im Endfaden nicht vorhanden. In einem Einzelfalle glaubte ich Andeutungen von indirekten Kerntheilungsfiguren vor mir zu haben. Es handelte sich um dieselben Gebilde, die Wit. in seiner Fig. 5 Tafel XX (diese Zeitschr. Bd. XLI 1885) am Übergange vom Endfaden in die Endkammer zeichnet, aher nicht hezeichnet. Jedoch, 
da ich ihnliche Figuren nicht wiedersah, auch sonst nichts fand, was auf indirekte Kerntheilung schließen ließ, halte ich auch die in Rede stehenden Gebilde nicht dafur. Vielleicht handelt es sich bei diesen Dingen um degenerirte Kerne, wofür die Struktur des Grenzgebietes zwischen Endkammer und Endfaden sprechen könnte. Um zusammenzufassen, so findet man Kerntheilungen im Endfaden wieder häufiger, als in der bindegewebigen Hüle. Sie sind amitotischer Art und gehen auf dieselbe Weise vor sich, wie es oben an anderer Stelle beschrieben wurde.

\section{H. Die Endkammer.}

Die Endkammer wurde bereits von Will und Konscmelt einem eingehenden Studium unterworfen. Erstgenannter Forscher giebt eine anschauliche Abbilduug der ganzen Endkammer in Fig. 3, Taf. XX seiner oft citirten Arbeit, während Konscnelt in den Fig. 84-95 der ebenfalls wiederholt angeführten Schrift Bruchstucke aus verschiedenen Gegenden der Endkammer zeichnet. Ich kann die Angaben der genannten Autoren zum großen Theil bestätigen und werde dem von ihnen Beschriehenen noch Einiges hinzuzufugen haben, entsprechend den etwas abweichenden Gesichtspunkten, von denen ich bei meiner Untersuchung ausging.

Das Innere der Endkammer wird von drei Zellbezirken eingenommen. Den hinteren Theil bildet das bereits abgehandelte Keimlager, daran schließt sich der Komplex der Nährzellen, welche nach vorn kleiner und kleiner werden. Am Gipfel endlich findet sich eine größere Menge gleichartiger Kerne. Diese letzteren schieben sich, wie Konschelt an Längsschnitten beobachtete, und ich bestätigen kann, keilfürmig in den die Mitte der Endkammer einnehmenden Komplex der Naihrzellen ein.

Zum größeren Theil wandelt sich nach dem genannten Autor diese an der Spitze der Endkammer gelegene Masse von Kernen in Nihrzellkerne um, während ein anderer Theil zurúckbleibt, ohne sich in Größe und Struktur zu indern. Solche Kerne findet man sowohl vereinzelt zwischen den großen Kernen im Centrum der Endkammer oberhalh) des freien Raumes, andererseits dicht an der Wand als eine zusammenbängende Lage, die sich vom obersten Gipfel der Endkammer bis zu deren Grunde erstreckt und hier in die kleinen Kerne des Keimlagers übergeht.

Die Grenzen der Zellen, welche diese an der Spitze der Endkammer gelegenen Kerne enthalten, lassen sich für gewöhnlich nicht erkennen, wie auch lionscuetr berichtet. In einem Einzelfalle jedoch waren sie 
mehr oder minder deutlich sichtbar und umschlossen polygonale Zellen.

Die Größe der in Rede stehenden Kerne nimmt nach hinten in der Endkammer allmählich zu. In dem Präparat, in welchem die Grenzen erkennbar waren, ließen die Kerne einen ziemlich breiten Rand fur das Protoplasma frei und fanden sich in diesen Zellen meist in der Einzahl, seltener zu zweien vor. Ihre Kontouren sind gut abgesetzt. Die Form der Kerne ist gewöhnlich rundlich; in anderen Fällen sind sie mehr in die Länge gestreckt und dann zuweilen mit Einschnurungen versehen. Der Chromatingehalt der Nuclei ist groß. In der Regel besitzen sie einen stark gefärbten und verschieden gestalteten Kernkörper, seltener einen in Durchschnürung begriffenen oder das Produkı davon, zwei Nucleolen.

Aus dem Vorkommen zweier Kerne in manchen Zellen und dem Verhalten, dass einzelne Kerne Einkerbungen aufweisen, lässt sich entnehmen, dass auch hier die Theilung nach dem Typus der Amitose vor sich gehe. Bezüglich derselben verweise ich, um nicht wiederholen zu müssen, auf meine für das Epithel gemachten Mittheilungen, da dic Vorgänge hier, wie dort, in recht übereinstimmender Weise wiederkehren.

Analog den für die jungeren Stadien der Epithelzellen maßgehenden Verhältnissen finden sich auch am Gipfel der Endkammer ziemlich häufig Mitosen (Fig. $29 a$ u. b), die den Amitosen ungefähr das Gleichgewicht halten. Jedoch muss dabei betont werden, dass das Vorkommen von Mitosen nicht regelmäßig hier zu konstatiren ist, sondern dieselben fehlen zuweilen gänzlich, wie ich das auch weiter oben schon für das Auftreten der Mitosen in anderen Theilen der Eiröhre hervorhob. In sehr seltenen Fällen wurden Mitosen auch im Centrum der Nährzellenpartie angetroffen, welches Verhalten aus dem fruher mitgetheilten, vereinzelten Vorkommen der kleinen Kerne in derselben erklärlich ist. Etwas häufiger kommen in der aus denselhen Kornen bestehenden Rundbekleidung der Endkammer Mitosen vor.

Es erubrigt noch den mittleren Abschnitt der Endkammer, nämlich die Nährzellenpartie zu besprechen.

Da Will eine "iußerst eingehende Beschreibung seiner "Oohlasten " gegeben, Korscuelt aber genau die Umwandlung der Nïhrzellen vom vorderen Theile bis zum Grunde der Endkammer geschildert hat, da endlich von beiden Autoren Ahbildungen dieser Verhältnisse vorliegen, werde ich mich in Rucksicht auf die Nährzellenpartic kurz fassen können. Immerhin ist es für das Verständnis des Ganzen nöthig, einen 
Üherblick uber die Strukturverhiltnisse der zelligen Elemente dieses Theiles der Endkammer zu geben.

Die Grenzen der Nährzellen sind mehr oder minder gut sichtbar, gewïhnlich treten sie am Rande der Endkammer deutlicher hervor. Die Zellen können einen, zwei und mehr Kerne enthalten (Fig. 30, 31 11. 32). Letztere nehmen von der Spitze gegen den Grund der Endkammer allmählich an Größe zu. Ihre Form ist, wie aus den Zeichnungen erhellt, verschieden. IIäufig sind sie rundlich oder oval, in anderen Fällen jedoch mehr in die Lïnge gestreckt und dann zuweilen mit Einschnúrungen versehen. Neben den gleich abzuhandelnden Kernkörpern besitzen sie einen großen Reichthum an Chromatin, das meistens in verschieden großen Brocken regellos durch den kern zerstreut ist. Häufig erkennt man in der Umgebung des Kernkörpers einen hellen Ring, indem hier die färbbare Substanz vom Kernkörper zurücktritt (Fig. 32).

Gewöhnlich findet sich nur ein verschieden gestalteter und stets stark gefärbter Nucleolus vor. Seltener trifft man in Zerschnürung begriffene (Fig. 32b) oder schon von einander getrennte Kernkörper (Fig. $30 b, 32 c u .(l)$ an. Mitunter kommen sogar drei Nucleolen vor.

Was den Theilungsvorgang anbetrifft, so kaun ich die Ausführungen meiner Vorgänger bestatigen, was ein Vergleich der Fig. 8-11 von WiLL und der Fig. 90 von Konscheıt mit meinen Abbildungen 30 und 31 auf den ersten Blick ergiebt. Erstgenannter Forscher beschreibt den Theilungsprocess seiner "Ooblasten" etwa folgendermaßen. Der Nucleolus streckt sich in die Länge und erleidet in der Mitte eine gelinde Einschnürung, die meist vom Kern nachgeahmt wird. Auf der zweiten Phase hat sich der Kernkörper in zwei Hälften getheilt, wïhrend der Kern zunächst noch auf dem Biskuitstadium verharrt. Das Endresultat dieses Processes stellen zwei dicht bei einander gelagerte, runde Kerne mit rundem Kernkörperchen dar. Hierbei hïlt sich das Protoplasma passiv. Diesen Kerntheilungsmodus konnte auch Konscnett bestitigen. Wie aus meiner Fig. 30 ersichtlich ist, befinde ich mich ganz im Einklange mit genannten Autoren. Der Kern $b$ in Fig. 30 entspricht der zweiten Phase von WiLl und seiner Fig. 10 b. Der Kern a in meiner Fig. 30 stellt das Endresultat einer solchen Theilung dar. Dagegen bildet der Kern $a$ in Fig. 30 eine auch friiher beim Epithel schon erwïhnte Ausnahme von dem gewöhnlichen Theilungsvorgang. Bei demselben setzte nämlich die beiderseitige Einkerbung friber ein, als der Durchschnürungsprocess am Kernkörper stattfand. Zellen mit drei Kernen sind in $a$ und $b$ der Fig. 31 abgebildet, wihrend $c$ in Fig. 31 eine solche mil funf Kernen illustrirt (cf. Korsculet Fig. 90 und Will Fig. 11). 
Diese Zellen mit mehreren Kiernen sind jedenfalls ähnlich aufzufassen, wie die durch Fragmentirung der Kíme entstehenden mehrkernigen Zellen, welche man verschiedentlich im Thierreich, z. B. im Knochen der Wirbelthiere, als sog. Riesenzellen findet.

Wie sich durchaus nicht alle Zellen der Endkammer, welche zwei oder mehrere Kerne enthalten, weiter theilen, so ist dies auch bei den zweikernigen Zellen des Follikelepithels nicht immer der Fall. Das außerordentlich verbreitete Vorkommen zweikerniger Zellen im Epithel der Lifächer beweist dies zur Genüge, zumal sich die zweikernigen Zellen auch noch in den ältesten Eifachern finden. Es scheint fast als ob dieser Zweikernigkeit der Epithelzellen, die sich mit dem Auftreten der Amitose eingebürgert hat, eine ganz besondere Bedeutung zukäme.

Wenngleich ich nun auch meinen beiden Gewährsmännern in den Angaben, dass das Protoplasma sich nicht mittheile, zumeist beipflichten muss, sah ich andererseits einige wenige Bilder, die hierzu nicht passen wollen. Die Zelle $a$ in Fig. 32 hat auf zwei gegenüberliegenden Punkten Einschnürungen erlitten, die ich als Anzeichen der bald vollendeten Theilung derselben, und wohl mit Recht, anspreche. Zudem wirkte das Bild im Präparat noch frappanter, als ich dies auf meiner Zeichnung wiederzugeben vermochte.

Zum Schluss sei noch erwähnt, dass mitotische Theilungen von Nïhrzellkernen nicht stattfinden. Auch für die Nährzellen der Endkammer gilt sonach, wie für verschiedene andere Partien des Ovariums, dass die Theilung der Kerne und Zellen auf amitolischem Wege vor sich geht.

\section{Untersuchnngen an einigen anderen Hemipteren.}

Die Untersuchung der Eiröhren einiger anderer Hemipteren, nämlich von Notonecta glauca, Iydrometra lacustris, Ranatra linearis, Reduvius personatus, Pyrrhocoris apterus ergahen im Großen und Ganzen dieselben Befunde, wie sie für Nepa cinerea eingehend beschrieben wurden. In Folge dessen dúrften die dort geschilderten Verhältnisse vielleicht für die Ilemipteren im Allgemeinen gelten.

Für die Eiröhren der genannten Formen erwiesen sich Chromosmiumessigsäure und Pikrinschwefelsäure als recht brauchbare Fixirungsmittel, während Sublimat weniger gute Bilder lieferte. Dic Untersuchung wurde in der gleichen Weise, wie bei Nepa vorgenommen, nämlich an den jüngeren Follikeln durch Schnitte, an den ailteren durch die Methode des Ablösens des Epithels vom Dotter.

Die Elemente der Ovarien der in Rede stehenden Thiere sind von 
Will, Wielowiesski und Konschelt so eingehend beschrieben und in Zeichnungen niedergelegt worden, dass ich nicht näher auf sie einzugehen brauche. Ich werde vielmehr nur diejenigen Verhälnisse abhandeln, die sich in Rucksicht auf die etwas anderen Gesichtspunkte meiner Arbeit ergeben. Jedoch kann ich mich auch hierbei kurz fassen, da der histologische Bau der Ovarien der genannten Formen, wie schon Eingangs erwihnt, demjenigen von Nepa fast in allen Sticken gleichkommt, und ich mich daher auf diese Form beziehen kann.

Um in der für Nepa gewählten Reihenfolge zu bleiben, finden sich im Epithel der älteren Follikel Zellen mit einem und andere mit zwei Kernen. Eingestreut in diese kommen mit ziemlicher Häufigkeit Kerne vor, die in der amitotischen Theilung begriffen sind. Letztere wird durch einen Zerschnürungsprocess am Kernkörper und dessen Zerfall in zwei Theilstucke eingeleitet. Was das quantitative Verhälnis der Zellen mit einem und andererseits mit zwei Kernen betrifft, so sei erwähnt, dass mit dem zunehmenden Alter der Follikel die Zellen mit zwei Kernen die Oberhand gewinnen. Besonders bemerkt sei noch, dass in den alteren Follikeln die Theilungen ausschließlich nach dem Typus der Amitose stattfinden.

Fur das Epithel der jüngeren Eifacher habe ich beide Arten der Theilung neben einander hergehend konstatiren können. Das für Nepa geschilderte wechselnde Vorkommen der mitotischen Figuren in den verschiedenen Eiröhren war auch für sämmtliche andere untersuchten Hemipteren festzustellen. Zuweilen fanden sich die in indirekter Theilung befindlichen Zellen ganz besonders häufig, während sie das andere Mal nur spärlich oder gar nicht vertreten waren.

Der entleerte Follikel unterliegt dem für Nepa ausführlich geschilderten Resorptions- und Degenerationsprocess.

Im Eiröhrenstiel theilen sich die Kerne nur auf amitotischem Wege.

In der Peritonealhulle liegt zumeist nur e in Kern in einer Zelle. Die Theilungen in derselben sind von verhältnismäßig seltenem Vorkommen und geschehen, so weit meine Untersuchungen reichen, nur direkt. Zwar konnte ich für Nepa neben den für gewöhnlich vorkommenden amitotischen Theilungen in drei Fällen mitotische Figuren nachweisen; ich verfügte aber auch uher ein bei Weitem größeres Beobachtungsmaterial von Nepa, als von den ubrigen Formen.

Auch im Keimlager gehen die Theilungen nach meinen Bcohachtungen in der Regel auf direktem Wege vor sich. Ich vermisste entgegen den Verhätnissen von Nepa, wo sich auch mitotische Figuren wenigstens in seltenen fällen fanden, dieselben im Keimlager von Notonecta und Reduvius gänzlich. Bei IIydrometra, Ranatra, Pyrrho- 
coris dagegen konnte ich sie einige wenige Male konstatiren, womit ich allerdings nicht sagen will, dass sie bei Notonecta und Reduvius gänzlich fehlten.

Die Elemente der den Gipfel der Endkammer einnehmenden Kernpartie können sich, wie für Nepa früher angegeben wurde, nach beiden Typen theilen. Auch hier muss ich wieder auf die schon für das jüngere Epithel erwähnte, wechselnde IIäufigkeit der mitotischen Figuren aufmerksam machen. Während bei den übrigen Formen die Zellgrenzen nicht zu erkennen waren, wiesen einige meiner Präparate von Pyrrhocoris dieselben scharf ausgepräigt auf.

In der Nährzellenpartie kommen entsprechend den fur Nepa geschilderten Verhältnissen reichliche Fragmentationen vor. Bis zu sechs Kerne beobachtete ich in einer Zelle. Von Besonderheiten möchte ich zunächst das bei Notonecta häufige Vorkommen zweier, ziemlich weit von einander entfernter Kerne in einer Zelle erwähnen, deren zugekehrten inneren Begrenzungen beträchtliche Vorsprunge zeigen. Leider war es mir nicht möglich, in Theilung begriffene Vorstufen solcher Kerne zu finden. Zweitens ist der fur Nepa charakteristische helle Hof um die Nucleolen der Nihrzellkerne auch bei Hydrometra gegenwärtig. Drittens sind bei manchen Eiröhren von Pyrrhocoris in den Nährzellkernen keine Kernkörper zu erkennen. Bei letztgenannter Form sah ich auch in einem Einzelfalle eine den Närzellkernen an Größe bedeutend nachstehende Mitose inmitten der Nährzellpartie (cf. das Entsprechende bei Nepa).

Während nun bei den Elementen des in Rede stehenden mittleren Theiles der Endkammer Zell theilung meist aushleibt, sah ich solche bei Notonecta, Reduvius und Pyrrhocoris doch zuweilen angedeutet, indem nämlich die Zellbegrenzung mehr oder minder tiefe Einschntirungen erlitten hatte.

Im Endfaden geschehen die mäßig häufigen Theilungen nur auf amitotischem Wege.

Aus den vorstehenden Erörterungen ergiebt sich eine große Ühereinstimmung der hier behandelten Verhältnisse der Ovarien bei sämmtlichen untersuchten Hemipteren. Es kann nicht bezweifelt werden, wie besonders aus den eingehender geschilderten Untersuchungen an Nepa hervorgeht, dass der Amitose, welche sich sowohl bei den jungsten, noch wenig zellenreichen und kleinen Eifächern, wie auch in den schon älteren Follikeln findet, eine wichtige Rolle bei der Vermehrung der Zellen zukommt. Auf diesen Punkt wird weiter unten bei den Schlussbetrachtungen noch eingegangen werden. Ein schlagender Be- 
weis fur die reiche Vermehrung der Zellen wiirde durch genaue Vergleichung der Zahl der Epithelzellen in jüngeren und älteren Eifollikeln zu geben sein. Meine Absicht, derartige Zïhlungen anzustellen, wurde leider dadurch verbindert, dass ich genöthigt war, meine Untersuchungen abzuschließen. Immerhin kann ich nach meinen Beobachtungen mit Sicherheit annehmen, dass zwischen den letzten Eifachern, in denen Mitosen reichlicher vorkommen und zwisehen den Endfollikeln der Eiröhre ein erheblicher Zahlenunterschied der Epithelzellen zu Gunsten der äteren Follikel besteht. Es ist selbstverständlich und braucht kaum erwähnt zu werden, dass ich bei der Ausführung meiner Untersuchungen stets darauf achtete, ob sich irgendwelche Beziehungen, vielleicht sogar Zwischenstufen, zwischen amitotischer und mitotischer Kerntheilung auffinden ließen, doch konnte ich bei den mir vorliegenden, zur Entscheidung dieser Frage offenbar sehr günstigen Objekten nichts Derartiges auffinden.

\section{Anhang.}

\section{Untersuchung an Locusta viridissima.}

Das konstante Vorkommen der Amitose in den Eierstöcken der Hemipteren legte es nahe, Ähnliches auch bei anderen Insekten zu vermuthen. Speciell war dabei an die Orthopteren zu denken, von denen Konschelt in seiner Abhandlung "über die Bildung der Eihallen etc." derartige Verhältnisse beobachtet hatte. Ich sammelte daher Material von verschiedenen Orthopteren, von denen ich jedoch nur Locusta viridissima bearbeiten konnte.

Die Konservirung, Färbung und sonstige Methode waren dieselben, wic sie bei den Ilemipteren angewandt wurden.

Jede Eiröhre von Locusta besteht aus einer Anzahl hinter einander liegender Follikel. Von diesen nehmen die vorderen nur allmählich von vorn nach hinten an Größe zu, während auf diese eines oder einige wenige bedeutend umfangreichere Eier folgen.

Im Epithel dieser letzteren, älteren Eifächer, welches eine mechanische Ablösung vom Dotter zulässt, findet sich nun im Gegensatz zu den Follikeln gleicher Stufe der Hemipteren fast immer nur e in rundlicher Kern in der Zelle. Die Anordnung des Chromatins spricht dafur, dass sich die Kerne im Ruhestadium befinden. Es sind keine Nucleolen vorhanden und die chromatische Substanz ist gleichmäßig über den ganzen Kern vertheilt. Dieser gewinnt hierdurch ein gekörneltes Aussehen. In ganz seltenen Fällen sah ich Kerne, die in amitotischer Theilung begriffen waren. Diese wenigen Ausnahmen kïnnen jedoch die 
Regel, nach der im Follikelepithel dieser Stadien wohl im Allgemeinen keine Theilungen mehr stattinden, nicht beeinträchtigen. Mitotische Figuren werden im Epithel dieser ilteren Eifächer üherhaupt nicht mehr gefunden.

Im Epithel der jüngeren Eifïcher, d. lı. derjenigen, welche den Bezirk vom Endfaden bis zu den soeben beschriebenen, umfangreichen Follikeln einnehmen, kommen reichliche mitotische Figuren aller Stadien vor. Dies bedeutet einen weiteren Unterschied gegenüber den Hemipteren, bei welchen die Mitosen in den Follikeln von ungefilhr gleichem Alter nicht mehr aufzufinden sind, mithin also nicht so weit in der Eiröhre herunterreichen. Bei den Hemipteren fand ich die Mitosen in verschiedenen Eiröhren in sehr differenter Zahl. Dies war bei Locusta nicht der Fall. Möglicherweise kann dieses Verhalten allerdings dadurch seine Erklärung finden, dass ich das Orthopterenmaterial innerhalb weniger Tage des Spätsommers einlegte, während die Hemiptereneiröhren zu verschiedenen Jahreszeiten gesammelt wurden. Wie schon für die Hemipteren hervorgehoben wurde, übertreffen auch hier die karyokinetischen Figuren die umliegenden Kerne an Größe und fallen leicht durch die dunkle Färbung und Anordnung der Chromosome auf.

Neben den Mitosen fand ich zumal im jüngeren Epithel auch direkte Kerntheilungen. Die amitotische Theilung kann auch hier durch einen Durchschnürungsprocess am Kernkörper und den Zerfall desselben in zwei Stücke eingeleitet werden. In anderen Fällen fehlen jedoch die Nucleolen und die Theilung besteht in Folge dessen in einer bloßen Durchschnủrung des Kernes. Das oftmals zu beohachtende Auftreten zweier Kerne in ein und derselben Zelle spricht nach den bei den IIemipteren gewonnenen Erfahrungen außer der direkten Beobachtung der amitotischen Theilung ebenfalls genügend für das Vorkommen derselben.

Im Endfaden, der häufig fibrillär gestreift er'scheint, kommen, um es gleich vorweg zu sagen, entsprechend den Verhältnissen der Hemipteren ke in e Mitosen vor. Dagegen finden sich hier und da ein- oder beiderseitig eingeschnürte Kerne mit zwei Nucleolen. Im Einklange mit meinen Befunden bei den Hemipteren muss ich daher annehmen, dass im Endfaden die Theilungen nur auf amitotischem Wege erfolgen und von mäßig häufigem Vorkommen sind.

In Vergleich mit den bei den Hemipteren beobachteten Verhiiltnissen ist von Locusta zu sagen, dass hier die Amitosen zwar ebenfalls ziemlich häufig vorkommen, aber nicht von so großer Verbreitung und Bedeutung sind, wie bei den Hemipteren. 
Es war meine Absicht die Verhältnisse der Kerntheilungen in den Ovarien auch noch bei anderen Orthopteren, sowie bei den Insekten mit mehrfachen Nährkammern, speciell bei Dytiscus, zu untersuchen, doch konnte ich leider das bereits für diese weiteren Studien zusammengebrachte Material nicht ausnutzen, da ich genöthigt war, meine Untersuchungen abzubrechen.

\section{Schlussbetrachtungen.}

Aus den geschilderten Beobachtungen geht hervor, dass die Amitose im Ovarium der untersuchten Hemipteren eine wichtige Rolle spielt. Sie findet sich in den jünsten Eifächern, die nur erst aus einer verhiiltnismäßig geringen Zahl von Zellen bestehen und in denen zweifelIos eine rege Zellvermehrung stattfindet. Weiterhin ist sie aber auch in den mittleren und älteren Eifollikeln häufig und fuhrt hier entweder, wie im ersteren Falle, zur Theilung der Zellen, oder zur Bildung zweikerniger Zellen. In den jüngeren Eifächern sind auch die Mitosen ziemlich häufig, wie gezeigt wurde. Hier findet also auch eine Vermehrung der Zellen auf mitotischem Wege statt. Bald jedoch treten die karyokinetischen Figuren zurück und fehlen schließlich ganz. Jetzt vollzieht sich die Theilung der Zellen nur noch auf amitotischem Wege. Trotzdem ist als zweifellos zu betrachten, dass die Zahl der Zellen noch in ganz erheblicher Weise zunimmt. Die amitotische Theilung ist also in dem vorliegenden Falle, in dem sicherlich eine größere Zahl von Generationen nach einander auf diese Weise gebildet wird, von Wichtigkeit. Es liegt ein Fall vor, in welchem die Amitose funktionelle Bedeutung beansprucht.

Derartige Erscheinungen sind wiederholt beschrieben worden. Wie schon Eingangs erwähnt wurde, liegt es nicht in meiner Absicht nach den erschöpfenden Darstellungen Fr.eming's auf eine Besprechung der Litteratur einzugehen, doch muss ich einige Fäle hier in meine Betrachtung ziehen, da sie mit den von mir beschriebenen Verhältnissen eine gewisse Übereinstimmung zeigen.

Nach Beocimaxy und Jonssox, besonders nach der Darstellung des letzteren Forschers, vollzieht sich in den Embryonalhúllen des Skorpions die amitotische Theilung auf eine Weise, dass man auch hier auf eine funktionelle Bedeutung derselben schließen darf, obwohl allerdings in diesem Gewebe die Zellen in nicht allzu langer Zeit der Vernichtung entgegengefuhrt werden, jedenfalls weit frither, als im Ovarialepithel der Insekten.

Aroold, Flemming, Knold, Loewit und andere Autoren beolachteten nehen der mitotischen auch die amitotische Kerntheilung in den Blut- 
zellen, und besonders der letztere Forscher ist eben so, wie nach ihm Knolt, geneigt, der Amitose eine Bedeutung für die fortgesetzte Vermehrung der Zellen d. h, ihr einen sogenanuten regenerativen Charakter zuzuschreiben.

Frenzel hält in verschiedenen Publikationen an der von anderer Seite (Zieglen, voм Ratu) bezweifelten Ansicht fest, dass in der Mitteldarmdruse des Flusskrebses amitotische Theilungen von durchaus funktioneller Bedeutung vorkommen.

Nach Garxox's älteren Beobachtungen hat die direkte Theilung in den Fettzellen und Matpigmischen Gefäen der Insekten, sowie in den Muskelzellen der Arthropoden so allgemein statt, dass ihr ebenfalls ein regenerativer Charakter zuzuerkennen ist.

Cuavs beobachtete in verschiedenen Geweben von Branchipus amitotische Theilungen und scheint denselben funktionelle Bedeutung beizumessen.

Es würde hier noch eine ganze Anzahl alterer und neuerer Äußerungen über die funktionelle Bedeutung der Amitose angeführt werden künnen, doch möchte ich nur auf die immer wiederkehrende Angabe von der bedeutungsvollen Rolle eingehen, welche der Amitose bei der Bildung der Samenzellen verschiedener Thiere zukommt.

Eine ganze Reihe von Autoren, ich nenne nur v. Bardelebex, Meves, Sanfelice, Venson, beobachteten die Amitose im Ifoden, besonders von Wirbelthieren, aber auch von Wirbellosen (Insekten), und nach ihren Befunden schien es, als ob dieser Vorgang wirklich zur Neubildung von Zellen fuhrte und von funktioneller Bedeutung sei. Dieser Ansicht stellten sich besonders Ziegler und vos Rati entgegen, indem Letzterer in den amitotisch sich theilenden Zellen unwesentliche Elemente sieht, welche nur das Stützgerüst des Hodens bilden, und im Übrigen im Anschluss an Ziegler betont, dass alle Zellen, welche amitotische Theilung erfuhren, sich mitotisch úberhaupt nicht mehr, amitotisch hüchstens nur noch wenige Male theilen können, dann aber zu Grunde gehen. Der Vertreter dieser letzteren Ansicht ist besonder's H. E. ZIEgLer, welcher scharf hervorhebt, dass die Amitose, wo sie auftritt, am Ende einer Reihe von Zelltheilungen steht und daher stets einen degenerativen Charakter hat. Dieser Ansicht vermag sich Furmмrvg in seiner neuesten Publikation nicht durchaus anzuschließen, zum mindesten meint er, dass diese Auffassung nicht mit genügender Sicherheit entschieden sei.

Obwohl mir beim Beginn meiner Untersuchungen nach dem Stand der damaligen Kenntnisse die Ansicht Ziegler's die größere Wahrscheinlichkeit für sich zu haben schien, so muss ich nach 
meinen eigenen Beobachtungen sagen, dass die von mir untersuchten Verhältnisse, sowie die Angaben einiger der oben angeführten Autoren darauf hinweisen, dass die amitotische Theilung doch bis zu einem gewissen Grade einen funktionellen Charakter trage oder besser gesagt, zur fortgesetzten Theilung von Zellen Anlass geben kann. Allerdings ist es ja richtig, dass theilweise in den von mir untersuchten Faillen das betreffende Gewebe, d. h. das Follikelepithel der Ovarien, zu Grunde geht, aber andererseits fand ich die amitotischen Theilungen auch im Endfaden, in der Endkammer, im Leitungsapparat (Eiröhrenstiel) und in der sogenannten Peritonealhúlle. In einigen dieser Gewebe kounte ich wohl amitotische Theilungen in Menge, niemals aber Mitosen auffinden, so dass letztere entweder ganz fehlen oder doch nur iußerst selten vorkommen. Was aber das Ovarialepithel anbetrift, so hob ich bereits oben hervor, dass die amitotischen Theilungen schon sehr früh und an ganz jungen Kernen sich finden, die von ihrer späteren Ausbildung und von ihrem endlichen Untergange noch weit entfernt sind. Auch bei ihnen kann also von einem degenerativen Charakter zunächst noch nicht gesprochen werden. Ich habe somit allen Grund zu der Annahme, dass in den Ovarien der Hemipteren eine ganze Reihe amitotischer Zelltheilungen auf einander folgt.

MI a rburg, 6. Februar 1895.

\section{Litteraturangaben.}

AnNoLD (87), Über Theilungsvorgänge an den Wanderzellen, ihre progressive und regressive Metamorphose, in: Arch, für mikr. Anat. Bd. XXX. 2. Heft. 1887.

v. Bardeleben (92), Über Spermatogenese bei Säugethieren, besonders beim Menschen. Verhandl, der anat. Gesellsch. Wien. 1892.

Blochann (85), Über direkte lierntheilung in der Embryonalhülle der Skorpione. Morphol. Jahrb. Bd. X. 1885.

Brader (93), Zur Kenntnis der Reifung des parthenogenetisch sich entwickelnden Eies von Artemia salina, in: Zool. Anz. 16. Jahrg. 1893.

Cannoy (S5), La cytodiérèse chez les Arthropodes. in: La Cellule. Louvain 1885.

Cinun $(90)$, Über die Bedeutung der direkten Kerntheilung. in : Sitzungsherichte der Physik.-Ökonom. Gesellsch. Königsberg 1890.

Craus (86), Arbeiten des zool. Instituts. Wien 1886.

Cornil, Sur le procédé de division indirecte des noyaux et des cellules epitheliales dans les tumeurs. Arch. de phys. norm, et path. 3 sér. T. VIII.

Finmang (82), Zellsubstanz, Kern und Zelltheilung. Leipzig 1882.

Flemaing (91), Über Theilung und kiernformen bei Leukocyten und iiber deren Attraktionssphären. in: Arch, f. mikr. Anat. Bd. XXXVII. 1891. 
Flemuing (92, 93), Zelle, Amitose. in : Ergebnisse der Anatomie und Entwicklungsgeschichte. Bd. II. 1892. Bd. III. 1893.

FoL (83), in : Archiv. des scienc. physiq. et natur. Genève. 15. Oct. 1883.

Frexzel (91), Zur Bedeutung der amitotischen (direkten) Kerntheilung. Biol. Centralblatt. Bd. XI. 1891.

Frenzel (93), Die Mitteldarmdrüse des Flusskrebses und die amitotische Zelltheilung. in: Arch. f. mikr. Anat. Bd. XLI. 1893.

IIansemann (91), Über pathol. Mitosen. Vinchow's Arch. Bd. CXXIII. 1891.

0. Hertwig (93), Die Zelle und die Gewebe. Jena, Fischer, 1893.

Hesse (94), Zur vergleichenden Anatomic der Oligochäten. Zeitschr. f. w. Zuol.

Bd. LXIII. 1894.

Hickson Sydey J. M. A. (94), The early stages in the development of Distichopora violacea, with a short Essay on the Fragmentation of the Nucleus. in:

The Quarterly Journal of microscopical Science. 1894.

Jonnsos (92), Amitosis in the embry onal envelopes of the scorpion. Bull, of the mus. of compar. Zoology, Harvard College. Vol. XXII, 3. 1892.

linol. (93), Über die Blutkürperchen bei wirbellosen Thieren. Sitzungsber. der Wiener Akad. d. Wiss. Math.-naturw. Klasse. Bd, CII. Abth. 3. 1893.

horschelt (86), Über Entstehung und Bedeutung der verschiedenen Zellenelemente des Insektenovariums, in : Zeitschr. f. w. Zool. Bd. XLII. Leipzig 1886.

Konschelt (87), Über einige interessante Vorgange bei der Bildung der Insekteneier. in: Zeitschr. f. w. Zool. Bd. XLV. Leipzig 1887.

Konschest (87), Über Bildung der Eihüllen, der Miliropylen und Chorionanhinge bei den Insekten, in: Halle 1887. 40. aus : Nova acta der Kais, Leop. Carol. Deutsch. Akademie. Bd. LI. Nr. 3.

Konscuelt (89), Beiträge zur Morphologie und Physiologie des Zellkerns. in: Zool. Jahrb. Bd. IV. 1889.

Loewit (90), Über Amitose. Centralblatt f. allgem. Path. und path. Anatomie. Bd. I 1890 .

LoEwit (91), Über Neubildung und Beschaffenheit der weißen Blutkürperchen, in: ZiEGLek's Beitr. Bd. X. 1891.

Loewit (91), Über amitotische kerntheilung. in: Biol. Centralblatt. Bd. IX. Nr. 17. 1891.

Mrves (91), Über amitotische Kerntheilung in den Spermatogonien des Salimanders. in: Anat. Anz. Nr. 22, 6. Jahrg. 1891.

voм Ratn (91), Über die Bedeutung der amitotischen Kerntheilung im Hoden. in : Zool. Anz. Nr. 393-395. 1891.

yom Raти (93), Bedeutung der Amitose in den Sexualzellen, in : Zeitschr. f. w. Zool. Bd. LVII. 1893.

SAnfelice (90), Intorno al modo di divisione delle Cellule germinale del testicolo. Sec. edizione Napoli. Casa editrice Car. Dott. V. Pasquale. R. Universití 1890.

Schottländer ( 88 ; Über Kern- und Zelltheilungsvorgïnge im Endothel der entziindeten Hornhaut. Arch, f. mikr. Anat. Bd. XXX1. 1888.

Strasburger (93), Zu dem jetzigen Stande der Kern- und Zelltheilungsfragen. in: Anat. Anz, 8. Jahrg. 1893.

VERSON (91), Zur Beurtheilung der amitotischen Kerntheilung. in: Biol. Centralbl. Bd. XI. 1891.

Wielowiejsk (86), Über den Bau des Insektenovariums. Krakau 1886. 
TVIL. (85), Bildungsgeschichte und morphologischer Werth des Eies von Nepa cinerea L. und Notonecta glauca L. in: Zeitschr. f.w. Zool. Bd, XLI. Leipzig 1885 .

Ziegler (91), Die biolog. Bedeutung der amitotischen Kerntheilung im Thierreich. in: Biol. Centralbl. Bd. IX. Nr. 12, 13, 15. Juli 1891.

Ziegler u, vom Ratu (91), Die anitotische Kerntheilung bei den Arthropoden. in Biol. Centralbl. Bd. IX. Nr.24. 1891.

\section{Erklärung der Abbildungen.}

Die Fig. 1-17 stellen Bruchstücke des Epithels älterer Eifächer dar, deren Dotter durch Abpinseln entfernt worden war. Die Fig. 18-32 wurden nach Schnitten augefertigt. Bei sämmtlichen Abbildungen wurde der Zeichenapparat verwendet. Mit Ausnahme der Fig. 10, 12 und 27, welche von Sublimatkonservirungen stammen, sind alle anderen nach Eiröhren gezeichnet, welche mit Chromosmiumessigsäure fixırt waren. Diejenigen Buchstaben der Figuren, die ohne Weiteres deutliche, aher im Text erwihnte Gestaltungsverhälnisse bezeichnen, sind nichl besonder's erklärt.

\section{Tafel XIX.}

Fig. 1. Epithelzelle mit Kern im Anfang der direkten Theilung. Vergr. 440.

Fis. 2 c $c$, dd zusammengehörige Zellen. Ein Kiern defekt und nicht mitgezeichnet. Vergr. 360 .

lïg. 3. Kern $b \mathrm{im}$ Anfange, $c$ in fortgeschrittener amitotischer Theilung. Vergr. 360 .

Fig. 4. Kern c mit in Durchschnürung begriffenem Nucleolus. Zellen $d d z u-$ sammengehörig. Vergr. 360.

Fig. 5. Kern $a$ hufeisenförmig. In den Kernen $c$ und $f$ hernkörper in Durchschnürung. Vergr. 440.

Fig. 6. Kern $b$ hufeisenförmig. Vergr. 360 .

Fig. 7. Die beiderseitig stark eingeschnürte Zelle $b$ beherbergt in der einen Hälfte einen ovalen, in der anderen einen zweiten, bereits wieder in Theilung begriffenen Kern. Vergr. 360 .

Fig. 8. Kerne $c, d, e$ in amitotischer Theilung. Vergr. 360.

Fig. 9. Zellen a a zusammengehörig. Vergr. 440.

Fig. 10. Sublimatkonservirung. Kerne heller, als das Protoplasma. Vergr. 440.

Fig. 11. Kern $c$ mit einseitig leicht eingeschnürtem Nucleolus. Kern $f$ in amitotischer Theilung. Vergr. 360.

Fig. 12. Sublimatkonservirung. Der langgestreckte liern a zeigt eine auf zwei gegenüberliegenden Punkten begonnene Einschnïrung. Vergr. 440.

Fig. 13. Kern $c$ hufeisenförmig. Vergr. 360.

\section{Tafel XX.}

Fig. 14. Konglomerat von Epithelzellen. Die Kierne $a, b, e, f, g, h$, in verschiedenen Stadien der direkten Theilung. Vergr. 440.

Fig. 15. e, Spaltkern. Die beiderseits eingeschnürte Zelle $f$ enthält in der 
cinen Hälfte zwei schon rollständig getheilte lierne, in der anderen einen in Theilung begriffenen. Vergr. 180.

Fig. 16. $a$, Lochkern; $b$, Spaltkern. Vergr. 640.

Fig. 17. Kerne $c$ und $d$ hufeisenförmig, $e$ von einer Seile her fast durchgeschnürt mit gezackten, inneren Begrenzungen. Vergr. 360 .

Fig. 18. Theil eines Schnittes durch das achte Eifach. Kern $a$ mit in Durchschnürung begriffenem kernkürper; $l, d, e$ in amitotischer Theilung. Das heimbläschen ist dem Epithel dicht angelagert. $f$ und g lierne, welche die chitinigen Strahlen absondern. Vergr. 145.

Figg. 19. Theil eines Schnittes durch das füntte Eifach, lierne $a, b, c$ in amitotischer Theilung, $d, e, f$ mitotische Figuren. Vergr. 600 .

Fig. 20. Stiick eines Schnittes durch das vierte Eifach. Kerne $a, b$, e in amitotischer Theilung, in $c$ und $f$ Nucleolen in Durchschnirung, $g$ und $i$ lierne mit zwei Nucleolen, $h$ mitotische Figur. Vergr. 800.

Fig. 21. Gruppe von Kiernen aus dem ersten Eifach. Zellgrenzen undeutlich. Liern $a$ beiderseitig eingeschnürt. Nucleolus in $b$ eingekerbt. Vergr. 1000.

Fig. 22. Einige Kerne aus dem Keimlager. Zellgrenzen deutlich. a beiderseitig eingeschnürter Kern. Vergr. 800.

Fig. 23. Kerne aus dem Keimlager. a in Theilung, l, und $l$ mit in Durchschniirung begriffenem Kernkörper, in $c$ deren zwei. Vergr. 1000.

Fig. 24. Kerne aus dem Keimlager. a eingeschnürt. Vergr. 800.

Fig. 25. Kerne aus dem lieimlager. Beiderseitig eingeschnürter kiern a hat den Farbstoff gegenuber den umgebenden kernen stäker angenommen. Vergr. soo.

Fig. 26. Kerne aus dem Keimlager. $a$ Theil eines Ernährungsstranges, $b$ beiderseitig eingeschnürter Kern, $c$ mit in Durchschnürung begriffenem Vucleolus, $d$ Kern mit zwei Kernkörpern. Vergr. 800.

Fig. 27. Gruppe von Zellen aus den Eiröhrenstiel. $a$ und $e$ in Theilung begriffene Kerne. Vergr. 800.

Fig. $28 a, 28 b, 28 c$. Eigenthümliche, sternförmige Gebilde, von denen das erstere auf der Scheidewand des fuinften und sechsten Eifaches lag. Vergr. 800, $1550,1000$.

Fig. 29. Gruppe von Kernen aus der Spitze der Endkammer. $a$ und $b$ mitotische Figuren. Vergr. 760.

Fig. 30. Nahrzellen aus der Endkammer. Kerne a und $b$ in amitotischer Theilung. Vergr. 640.

Fig. 31. In den Zellen $a$ und $b$ drei, in Zelle $c$ fünf Kerne. Vergr. 640.

Fig. 32. Nährzellen vom Grunde der Endkammer. Zelle a hat auf zwei gegenüberliegenden Punliten Einschnürungen erlitten. Kern $b$ mit einseitigg eingeschniirtem Nucleolus. Vergr. 440. 
Druck von Breitkopf \& Ifärtel in Leipzig. 


\section{Lebenslanf.}

Ich, Henrici Emil Fraxz Precsse wurde am 2. Oktober 1867 zu Braunschweig (Herzogthum Braunschweig', geboren. Mein Vater war damals Kaufmann, spïter lebte er als Rentner, jetzt ist er todt. Ich bekenne mich zur lutherischen Religion. Nach dem Besuche einer Volksschule meiner Vaterstadt siedelte ich auf das dortige humanistische Gymnasium über, auf dem ich bis zur Oberprima verblieb. Um mich dem Studium der Thierheilkunde $z u$ widmen besuchte ich in den Jahren 1887-1891 nach einander die thierärztlichen Hochschulen zu Hannover, Stuttgart, München und Berlin. An erstgenannter Bildungsanstalt bestand ich das Tentamen physicum, an letzterer erhielt ich im Sommer 1891 die Approbation. Wiahrend meines Aufenthalts in Berlin war ich gleichzeitig zwei Semester an der FriedrichWilhelms Universität immatrikulirt. Yom 1. Oktober 1891 bis dahin 1892 genügte ich meiner Dienstpflicht beim kgl. bayr. I. Llanen-Regiment in Bamberg und leistete daranschließend eine achtwöchentliche Übung ab. Nach einer Wirksamkeit rom 1. Januar 1893 bis 1. April desselben Jahres als Assistent am Göttinger Thierarznei-Institut, in welche Zeit auch meine Beförderung zum kgl. baỵ. Veterinärarzt 2. Klasse der Reserve failt, bezog ich die Universitït Marburg. Dort betrieb ich naturwissenschaftliche, insbesondere zoologische Studien vier Semester hindurch.

Außer meinen thierärztlichen Vorlesungen und Kliniken hörte ich in Berlin die Vorlesungen des Herrn Privatdocenten Dr. Konscilect, in Göltingen die des Ilerrn Privatdocenten Dr. Büngen, in Marburg die Vorlesungen und Practica der Herren Professoren Dr. Melde, Benguaxi, Conex, A. Mexer, Konscuelt, Natone, Feussner, Kohl und des Herrn Privatdocenten Dr. Brauer.

Allen diesen Ilerren spreche ich hiermit meinen wïrmsten Dank aus. 



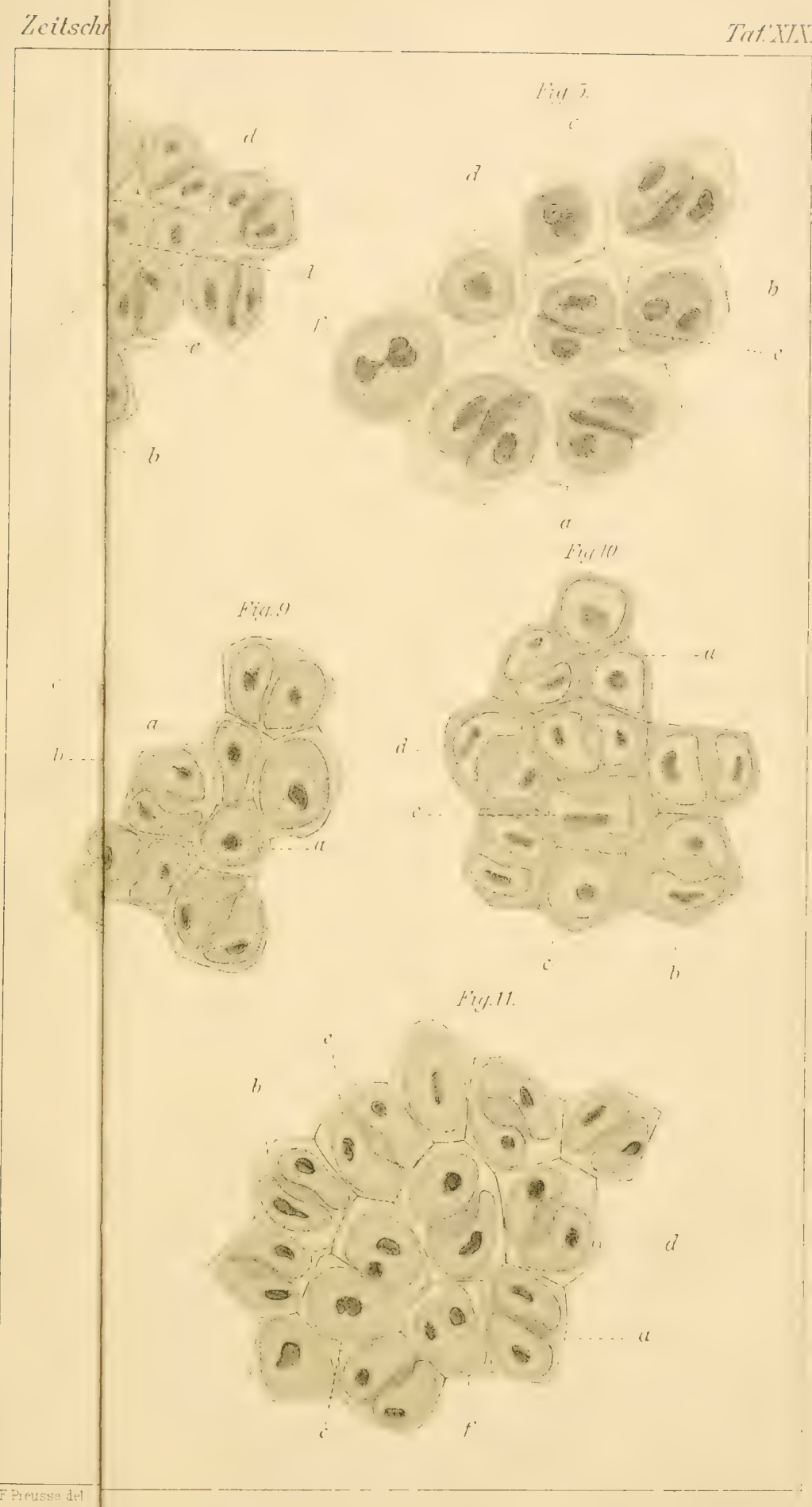





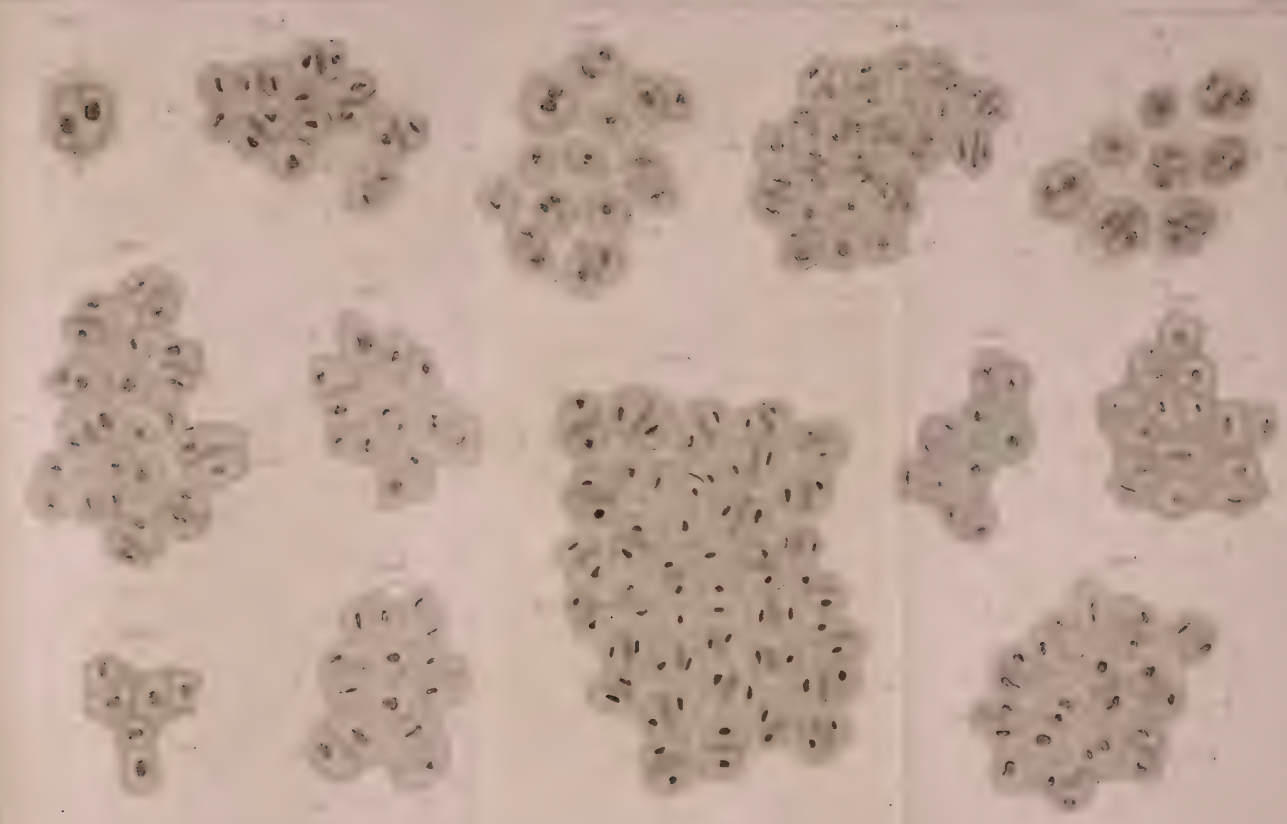



Zcilsthrith

a

है:

6.7

Iiv $: \because$

Fi! (...)

Fis...

fiv -rere

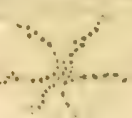





QL Preusse, Franz.

524.2 Die amitotische Kernthei-?iP7 lung in den Ovarien der He-leEnt. miptera. 
SMITHSONIAN INSTITUTION LIBRARIES 